CRYSTALLOGRAPHIC COMMUNICATIONS

ISSN 2056-9890

\section{Comparison of molecular structures of cis-bis[8-(dimethylphosphanyl)quinoline]nickel(II) and -platinum(II) complex cations}

\author{
Masatoshi Mori ${ }^{\mathrm{a}}$ and Takayoshi Suzuki ${ }^{\mathrm{b} *}$ \\ ${ }^{a}$ Graduate School of Natural Science and Technology, Okayama University, Okayama, 700-8530, Japan, and ${ }^{\mathbf{b}}$ Research \\ Institute for Interdisciplinary Science, Okayama University, Okayama, 700-8530, Japan. *Correspondence e-mail: \\ suzuki@okayama-u.ac.jp
}

Received 19 October 2020

Accepted 29 October 2020

Edited by S. Parkin, University of Kentucky, USA

The crystal structures of the complexes (SP-4-2)-cis-bis[8-(dimethylphosphanyl)quinoline- $\left.\kappa^{2} N, P\right]$ nickel(II) bis(perchlorate) nitromethane monosolvate, $\left[\mathrm{Ni}\left(\mathrm{C}_{11} \mathrm{H}_{12} \mathrm{NP}\right)_{2}\right]\left(\mathrm{ClO}_{4}\right)_{2} \cdot \mathrm{CH}_{3} \mathrm{NO}_{2}$ (1), and (SP-4-2)-cis-bis[8-(dimethylphosphanyl)quinoline- $\left.\kappa^{2} N, P\right]$ platinum(II) bis(tetrafluoroborate) acetonitrile monosolvate, $\left[\mathrm{Pt}\left(\mathrm{C}_{11} \mathrm{H}_{12} \mathrm{NP}\right)_{2}\right]\left(\mathrm{BF}_{4}\right)_{2} \cdot \mathrm{C}_{2} \mathrm{H}_{3} \mathrm{~N}$ (2), are reported. In both complex cations, two phosphanylquinolines act as bidentate $P, N$-donating chelate ligands and form the mutually cis configuration in the square-planar coordination geometry. The strong trans influence of the dimethylphosphanyl donor group is confirmed by the $\mathrm{Ni}-\mathrm{N}$ bond lengths in $\mathbf{1}, 1.970$ (2) and 1.982 (2) $\AA$ and, the $\mathrm{Pt}-\mathrm{N}$ bond lengths of $2,2.123$ (4) and 2.132 (4) $\AA$, which are relatively long as compared to those in the analogous 8-(diphenylphosphanyl)quinoline complexes. Mutually cis-positioned quinoline donor groups would give a severe steric hindrance between their ortho-H atoms. In order to reduce such a steric congestion, the $\mathrm{Ni}^{\mathrm{II}}$ complex in $\mathbf{1}$ shows a tetrahedral distortion of the coordination geometry, as parameterized by $\tau_{4}=0.199(1)^{\circ}$, while the $\mathrm{Pt}^{\mathrm{II}}$ complex in 2 exhibits a typical square-planar coordination geometry $\left[\tau_{4}=\right.$ $\left.0.014(1)^{\circ}\right]$ with a large bending deformation of the ideally planar $\mathrm{Me}_{2} \mathrm{Pqn}$ chelate planes. In the crystal structure of $\mathbf{2}$, three $\mathrm{F}$ atoms of one of the $\mathrm{BF}_{4}{ }^{-}$ anions are disordered over two sets of positions with refined occupancies of 0.573 (10) and $0.427(10)$.

\section{Chemical context}

8-Quinolylphosphanes are an intriguing class of ligands because they form a planar asymmetrical five-membered chelate ring via coordination through a phosphane-P atom having a strong $\sigma$-donating character and an imine-N atom incorporated in a $\pi$-conjugated quinoline ring (Salem \& Wild, 1992; Scattolin et al., 2017; Cai et al., 2018). The electronic properties of these ligands, in particular their $\pi$-bonding nature, may stabilize unusual electronic states of their transition-metal complexes (Suzuki et al., 1995; Hashimoto et al., 2010; Hopkins et al., 2019). In addition, the steric requirement from the planar quinoline moiety often has a significant influence on the properties of their metal complexes. For example, the nickel(II), palladium(II) and platinum(II) complexes containing two 8-(diphenylphosphanyl)quinoline $\left(\mathrm{Ph}_{2} \mathrm{Pqn}\right)$ in the $\operatorname{cis}(P, P)$ configuration exhibit a severe distortion of the square-planar coordination geometry around $M^{\mathrm{II}}(M=\mathrm{Ni}, \mathrm{Pd}$ or Pt; Suzuki, 2004; Hashimoto et al., 2010; Mori et al., 2020). The dimethylphosphanyl analogue, 8-(dimethylphosphanyl)quinoline $\left(\mathrm{Me}_{2} \mathrm{Pqn}\right)$, is an interesting derivative, because it would give a stronger trans influence, 
which could affect the steric congestion between the intramolecular ligands. However, the transition-metal complexes bearing $\mathrm{Me}_{2} \mathrm{Pqn}$ are limited to only those listed in section 4: Database survey, all of which were reported by our group. In 1995 we reported the preparation and crystal structure of ( $S P$ 4-2)- $\left[\mathrm{Pd}\left(\mathrm{Me}_{2} \mathrm{Pqn}\right)_{2}\right]\left(\mathrm{BF}_{4}\right)_{2}$ (Suzuki et al., 1995), but the crystal structures of the corresponding $\mathrm{Ni}^{\mathrm{II}}$ and $\mathrm{Pt}^{\mathrm{II}}$ complexes were not compared.

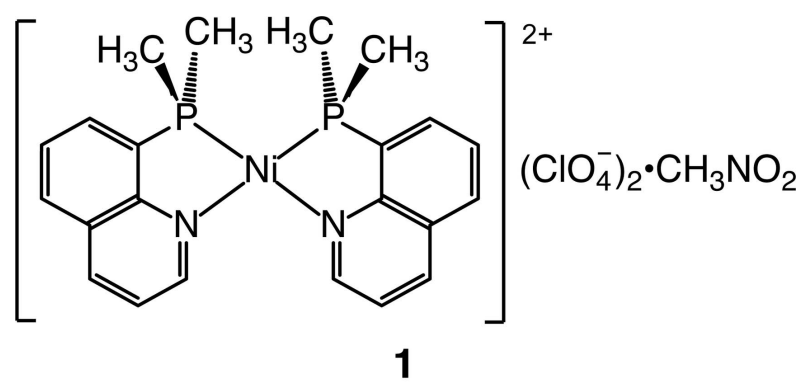

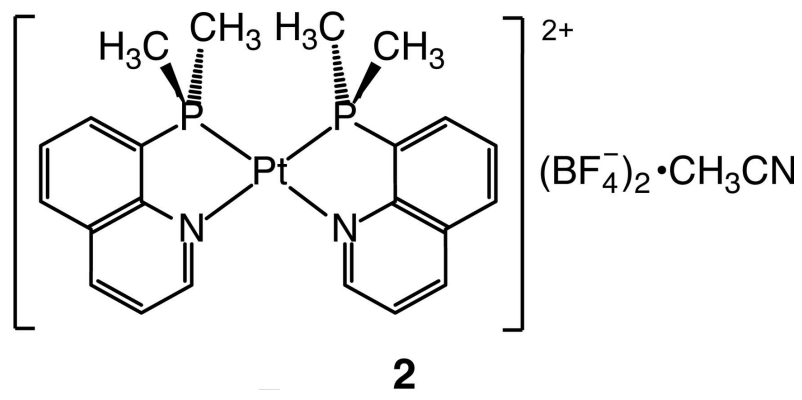

\section{Structural commentary}

A red block-shaped crystal of the $\mathrm{Ni}^{\mathrm{II}}$ complex, $\left[\mathrm{Ni}\left(\mathrm{Me}_{2} \mathrm{Pqn}\right)_{2}\right]\left(\mathrm{ClO}_{4}\right)_{2} \cdot \mathrm{CH}_{3} \mathrm{NO}_{2} \quad$ (1), recrystallized from nitromethane/diisopropyl ether and a colorless platelet crystal of the $\mathrm{Pt}^{\mathrm{II}}$ complex, $\left[\mathrm{Pt}\left(\mathrm{Me}_{2} \mathrm{Pqn}\right)_{2}\right]\left(\mathrm{BF}_{4}\right)_{2} \cdot \mathrm{CH}_{3} \mathrm{CN}(2)$, recrys-

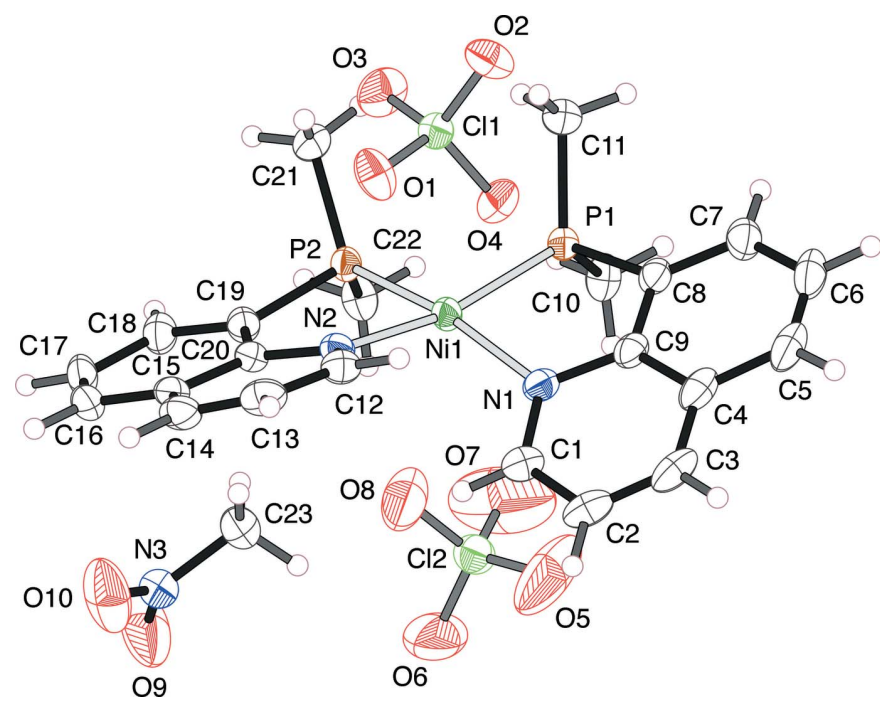

Figure 1

An ellipsoid plot of the molecular structures in $\left[\mathrm{Ni}\left(\mathrm{Me}_{2} \mathrm{Pqn}\right)_{2}\right]-$ $\left(\mathrm{ClO}_{4}\right)_{2} \cdot \mathrm{CH}_{3} \mathrm{NO}_{2}$ (1), showing the atom-numbering scheme, with ellipsoids drawn at the $50 \%$ probability level.
Table 1

Selected geometric parameters $\left(\AA,^{\circ}\right)$ for $\mathbf{1}$.

\begin{tabular}{lrlr}
\hline Ni1-N1 & $1.970(2)$ & Ni1-P2 & $2.1534(7)$ \\
Ni1-N2 & $1.982(2)$ & Ni1-P1 & $2.1576(7)$ \\
N1-Ni1-N2 & $97.01(9)$ & N1-Ni1-P1 & $86.13(7)$ \\
N1-Ni1-P2 & $166.27(7)$ & N2-Ni1-P1 & $165.68(6)$ \\
N2-Ni1-P2 & $85.97(6)$ & P2-Ni1-P1 & $94.29(3)$ \\
& & & \\
Ni1-P1-C8-C9 & $14.54(19)$ & Ni1-P2-C19-C20 & $18.86(19)$ \\
Ni1-N1-C9-C8 & $-11.8(3)$ & Ni1-N2-C20-C19 & $-9.7(3)$ \\
\hline
\end{tabular}

Table 2

Selected geometric parameters $\left(\AA,^{\circ}\right)$ for $\mathbf{2}$.

\begin{tabular}{lrlr}
\hline Pt1-N1 & $2.123(4)$ & Pt1-P2 & $2.2293(12)$ \\
Pt1-N2 & $2.132(4)$ & Pt1-P1 & $2.2365(12)$ \\
& & & \\
N1-Pt1-N2 & $97.13(15)$ & N1-Pt1-P1 & $82.76(11)$ \\
N1-Pt1-P2 & $178.51(11)$ & N2-Pt1-P1 & $179.55(11)$ \\
N2-Pt1-P2 & $81.93(11)$ & P2-Pt1-P1 & $98.17(4)$ \\
& & & \\
Pt1-P1-C8-C9 & $15.5(3)$ & Pt1-P2-C19-C20 & $21.2(4)$ \\
Pt1-N1-C9-C8 & $-18.9(5)$ & Pt1-N2-C20-C19 & $-19.0(5)$ \\
\hline
\end{tabular}

tallized from acetonitrile/diisopropyl ether were used for the $\mathrm{X}$-ray diffraction analysis.

In the crystal structure of $\mathbf{2}$, three $\mathrm{F}$ atoms of one of the $\mathrm{BF}_{4}{ }^{-}$anions show disorder over two sets of positions: (F2A, $\mathrm{F} 3 A$ and $\mathrm{F} 4 A)$ and $(\mathrm{F} 2 B, \mathrm{~F} 3 B$ and $\mathrm{F} 4 B)$. The occupancy parameters of these atoms were refined with suitable restrictions and found to be 0.573 (10) and 0.427 (10) for the $A$-set atoms and the $B$-set atoms, respectively.

In both crystals, two $\mathrm{Me}_{2} \mathrm{Pqn}$ ligands coordinate to a metal(II) center in the bidentate $\kappa^{2} P, N$ mode to form a cisisomer of the complex dication, $(S P-4-2)-\left[M\left(\mathrm{Me}_{2} \mathrm{Pqn}\right)_{2}\right]^{2+}(M$

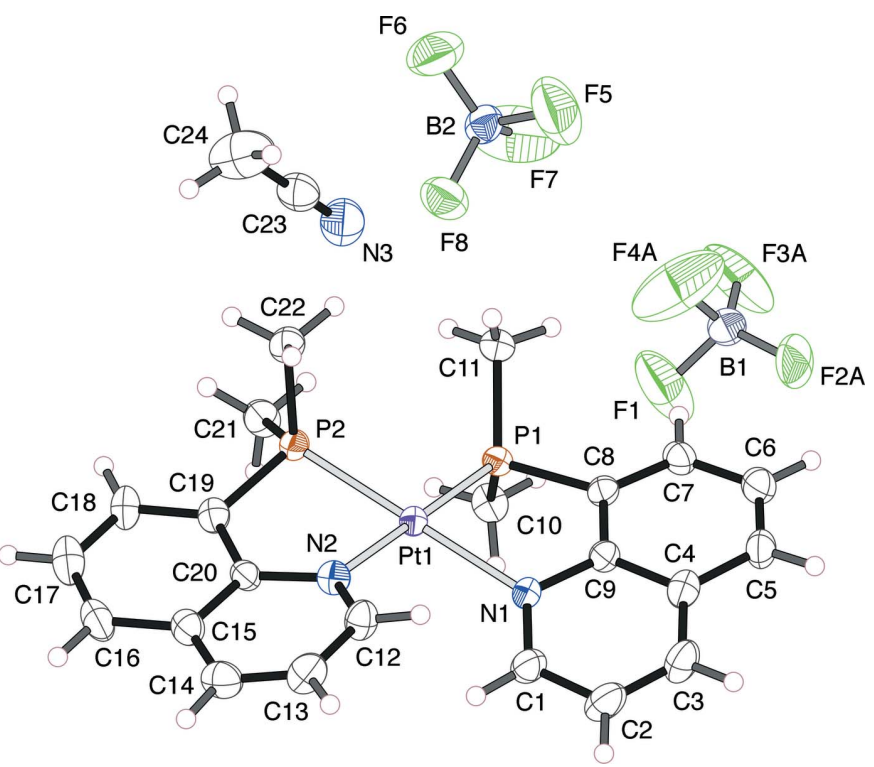

Figure 2

An ellipsoid plot of the molecular structures in $\left[\mathrm{Pt}\left(\mathrm{Me}_{2} \mathrm{Pqn}\right)_{2}\right]\left(\mathrm{BF}_{4}\right)_{2} \cdot-$ $\mathrm{CH}_{3} \mathrm{CN}$ (2), showing the atom-numbering scheme, with ellipsoids drawn at the $50 \%$ probability level. The minor component atoms (F2B, F3B and $\mathrm{F} 4 B$ ) of the positionally disordered $\mathrm{F}$ atoms are omitted for clarity. 


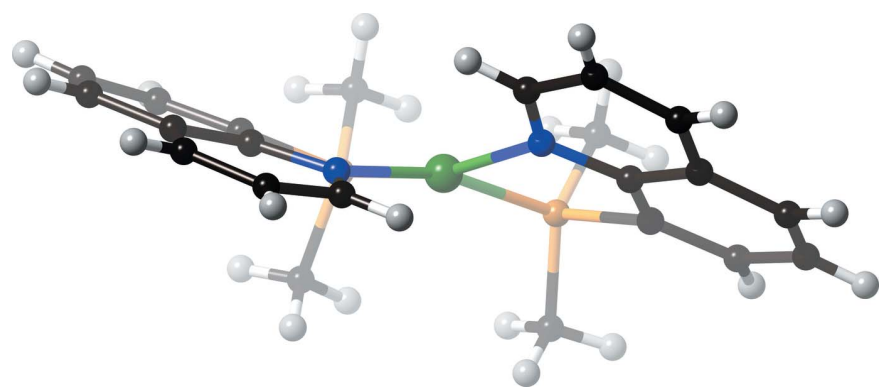

Figure 3

A perspective side view (from one of the NiPN coordination planes) of $\left[\mathrm{Ni}\left(\mathrm{Me}_{2} \mathrm{Pqn}\right)_{2}\right]\left(\mathrm{ClO}_{4}\right)_{2} \cdot \mathrm{CH}_{3} \mathrm{NO}_{2}$ (1). Color code: $\mathrm{Ni}$, dark green; $\mathrm{P}$, orange; $\mathrm{N}$, blue; $\mathrm{C}$, black and $\mathrm{H}$, gray.

$=\mathrm{Ni}$ or $\mathrm{Pt}$ ), having a roughly square-planar coordination geometry (Figs. 1 and 2, Tables 1 and 2). For the group 10 metal(II) complexes bearing 8-quinolylphosphanes, it was revealed that most of the $\operatorname{bis}\left(\kappa^{2} P, N\right)$-type complexes have a similar geometrical structure, except for those of the halide complexes (Suzuki, 2004; Mori et al., 2020), because the strong trans influence of the phosphane donor groups makes the mutually $\operatorname{trans}(P, P)$ configuration thermodynamically unstable. The $\mathrm{Ni}-\mathrm{N}$ bond lengths in $\mathbf{1}$ are $1.970(2)$ and $1.982(2) \AA$, which are slightly longer than those in $\left[\mathrm{Ni}(\mathrm{MePhPqn})_{2}\right]\left(\mathrm{BF}_{4}\right)_{2} \quad[\mathrm{MePhPqn}=8$ - $($ methylphenylphosphanyl)quinoline; $1.954(3)$ and $1.977(3) \AA]$ and $\left[\mathrm{Ni}\left(\mathrm{Ph}_{2} \mathrm{Pqn}\right)_{2}\right]\left(\mathrm{BF}_{4}\right)_{2}$ [1.954 (6) and $\left.1.949(5) \AA\right]$ (Hashimoto et al., 2010), indicating the trans influence becomes stronger in the order of $\mathrm{Ph}_{2} \mathrm{Pqn}<\mathrm{MePhPqn}<\mathrm{Me}_{2} \mathrm{Pqn}$. In the case of $\mathrm{Pt}^{\mathrm{II}}$ complexes, the $\mathrm{Pt}-\mathrm{N}$ bond lengths in 2 [2.123 (4) and $2.132(4) \AA]$ are similarly long, as compared to those in $\left[\mathrm{Pt}\left(\mathrm{Ph}_{2} \mathrm{Pqn}\right)_{2}\right]\left(\mathrm{ClO}_{4}\right)_{2}[2.107$ (4) and $2.108(5) \AA$; Mori et al., 2020]. By contrast, the $\mathrm{Ni}-\mathrm{P}$ bond lengths and the $\mathrm{P}-\mathrm{Ni}-\mathrm{N}$ chelate bite angles are comparable among the complexes $\mathbf{1}$ [2.1576 (7) and $2.1534(7) \AA ; 86.13(7)$ and $85.97(6)^{\circ}$ ], $\left[\mathrm{Ni}(\mathrm{MePhPqn})_{2}\right]\left(\mathrm{BF}_{4}\right)_{2} \quad[2.151(1)$ and $2.162(1) \AA ; 87.4(1)$ and $\left.86.6(1)^{\circ}\right]$ and $\left[\mathrm{Ni}\left(\mathrm{Ph}_{2} \mathrm{Pqn}\right)_{2}\right]\left(\mathrm{BF}_{4}\right)_{2} \quad[2.168(2)$ and 2.177 (2) $\AA ; 86.6$ (1) and $\left.84.6(1)^{\circ}\right]$. The $\mathrm{Pt}-\mathrm{P}$ bond lengths and the $\mathrm{P}-\mathrm{Pt}-\mathrm{N}$ bite angles in 2 [2.2293(12) and 2.2365 (12) $\AA ;$; 82.76 (11) and $81.93(11)^{\circ}$ ] are also comparable to those in $\left[\mathrm{Pt}\left(\mathrm{Ph}_{2} \mathrm{Pqn}\right)_{2}\right]\left(\mathrm{ClO}_{4}\right)_{2} \quad[2.2311(14)$ and 2.2318 (14) $\AA$; 83.29 (13) and $82.79(13)^{\circ}$ ].

Comparison of the $\mathrm{Ni}^{\mathrm{II}}$ complex cation in $\mathbf{1}$ and the corresponding $\mathrm{Pt}^{\mathrm{II}}$ complex cation in $\mathbf{2}$ shows an obvious

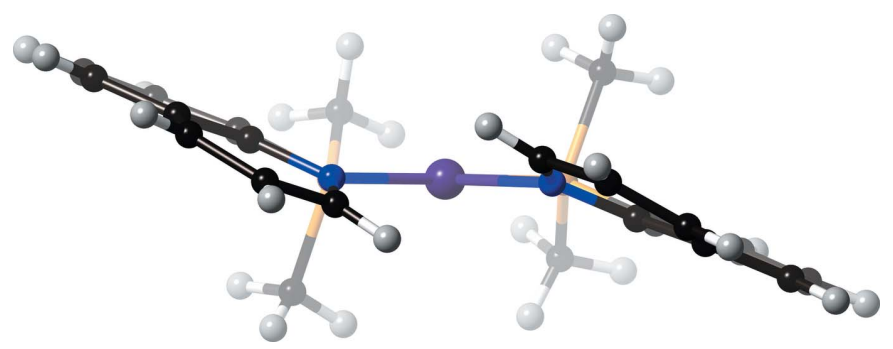

Figure 4

A perspective side view (from one of the PtPN coordination planes) of $\left[\mathrm{Pt}\left(\mathrm{Me}_{2} \mathrm{Pqn}\right)_{2}\right]\left(\mathrm{BF}_{4}\right)_{2} \cdot \mathrm{CH}_{3} \mathrm{CN}$ (2). Color code: $\mathrm{Pt}$, purple; $\mathrm{P}$, orange; $\mathrm{N}$, blue; $\mathrm{C}$, black and $\mathrm{H}$, gray.

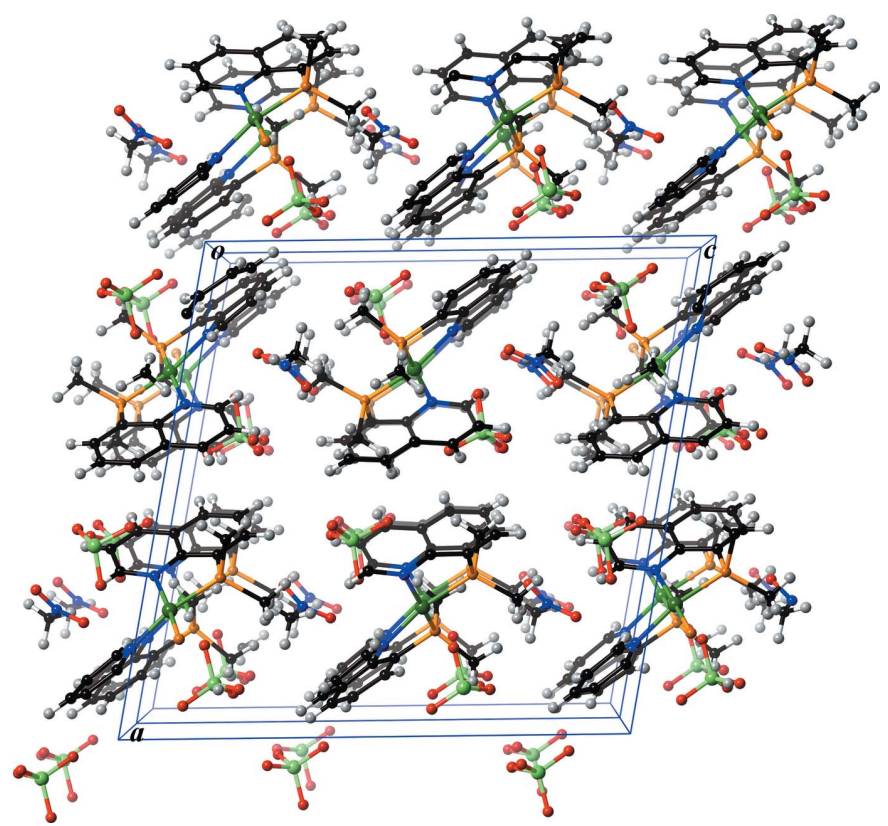

Figure 5

A packing drawing of $\left[\mathrm{Ni}\left(\mathrm{Me}_{2} \mathrm{Pqn}\right)_{2}\right]\left(\mathrm{ClO}_{4}\right)_{2} \mathrm{CH}_{3} \mathrm{NO}_{2}$ (1) along the crystallographic $b$ axis (two unit cells are shown). Color code: $\mathrm{Ni}$, dark green; $\mathrm{Cl}$, light green; $\mathrm{P}$, orange; $\mathrm{O}$, red; $\mathrm{N}$, blue; $\mathrm{C}$, black and $\mathrm{H}$, gray.

difference in their coordination geometry (Figs. 3 and 4). The four-coordinate $\mathrm{Ni}^{\mathrm{iI}}$ center in $\mathbf{1}$ exhibits a large tetrahedral distortion, as indicated by the $\tau_{4}$ value (Yang et al., 2007) of $0.199(1)^{\circ}$. This is due to the steric requirement from the planar quinoline moiety located in the mutually cis positions around the $\mathrm{Ni}^{\mathrm{II}}$ center. In the analogous MePhPqn and $\mathrm{Ph}_{2} \mathrm{Pqn}$ complexes, the $\tau_{4}$ values are $0.273(1)^{\circ}$ and $0.189(2)^{\circ}$, respectively. By contrast, the $\tau_{4}$ value of the $\mathrm{Pt}^{\mathrm{II}}$ complex in $\mathbf{2}$ is only $0.014(1)^{\circ}$, indicating a nearly perfect planar coordination geometry around the $\mathrm{Pt}^{\mathrm{II}}$ center. The corresponding value in $\left[\mathrm{Pt}\left(\mathrm{Ph}_{2} \mathrm{Pqn}\right)_{2}\right]\left(\mathrm{BF}_{4}\right)_{2}$ is $0.149(2)^{\circ}$ (Mori et al., 2020). It is obvious that the present planar structure of the $\mathrm{Pt}^{\mathrm{II}}$ center in 2 is a rare example. In this complex, the interligand steric interaction expected for the mutually cis-positioned quinoline groups could be reduced by envelope-type bending of the planar $\mathrm{Me}_{2} \mathrm{Pqn}$ chelate coordination, that is, the displacement of the $\mathrm{Pt}^{\mathrm{II}}$ metal center from the ideal plane defined by the chelate ring of 8-quinolylphosphanes. The dihedral angle, $\varphi_{\mathrm{C}}$, between the $[\mathrm{Pt}, \mathrm{P}, \mathrm{N}]$ coordination plane and the $[\mathrm{PCCN}]$ phosphanylquinoline planes in $\mathbf{2}$ are $21.53(16)$ and $24.76(16)^{\circ}$, and the displacement of the Pt1 atom from the ideal quinoline $\left[\mathrm{C}_{9} \mathrm{H}_{6} \mathrm{~N}\right]$ planes is $0.579(5)$ and $0.550(5) \AA$. The two quinoline planes are nearly parallel, with the dihedral angle between them being only $7.99(10)^{\circ}$. Such a synchronized bending deformation of two chelate coordination (Fig. 4) acts to reduce the steric congestion effectively. The corresponding $\varphi_{\mathrm{C}}$ values for $\mathbf{1}$ are 17.44 (9) and $19.76(9)^{\circ}$, and the dihedral angle between the two quinoline planes is obviously large, at $33.35(6)^{\circ}$. Interestingly, the analogous palladium(II) complex, $\left[\mathrm{Pd}\left(\mathrm{Me}_{2} \mathrm{Pqn}\right)_{2}\right]\left(\mathrm{BF}_{4}\right)_{2}$, has a $\tau_{4}$ value of $0.096(2)^{\circ}$ (Suzuki et al., 1995), which is in between those of the present $\mathrm{Ni}^{\mathrm{II}}$ and $\mathrm{Pt}^{\mathrm{II}}$ complexes. 


\section{Supramolecular features}

In the crystal structure of $\mathbf{1}$, there are two $\mathrm{ClO}_{4}{ }^{-}$anions and a $\mathrm{CH}_{3} \mathrm{NO}_{2}$ solvent molecule, in addition to the $\left[\mathrm{Ni}\left(\mathrm{Me}_{2} \mathrm{Pqn}\right)_{2}\right]^{2+}$ complex cation in the asymmetric unit. The asymmetric unit of the $\mathrm{Pt}^{\mathrm{II}}$ complex, 2, contains a $\left[\mathrm{Pt}\left(\mathrm{Me}_{2} \mathrm{Pqn}\right)_{2}\right]^{2+}$ complex cation, two $\mathrm{BF}_{4}{ }^{-}$anions (in one of which the positions of three F atoms are disordered) and a $\mathrm{CH}_{3} \mathrm{CN}$ solvent molecule. In the crystal structures of both $\mathbf{1}$ and $\mathbf{2}$ (Figs. 5 and 6, respectively), no remarkable intermolecular stacking or hydrogen-bonding interactions are observed.

\section{Database survey}

Metal complexes containing $\mathrm{Me}_{2} \mathrm{Pqn}$ have been reported by us, e.g., cis- $\left[\mathrm{Pd}\left(\mathrm{Me}_{2} \mathrm{Pqn}\right)_{2}\right]\left(\mathrm{BF}_{4}\right)_{2}$ (refcode ZIFPUZ in the CSD database, version 5.41, last update May 2020; Groom et al., 2016) and $\left[\mathrm{Pd}_{2} \mathrm{Cl}_{2}\left(\mathrm{Me}_{2} \mathrm{Pqn}\right)_{2}\right]$ (ZIFQAG; Suzuki et al., 1995), $\left[\mathrm{Cu}\left(\mathrm{Me}_{2} \mathrm{Pqn}\right)_{2}\right] \mathrm{PF}_{6}$ (OZILAL; Suzuki et al., 2011), $\left[\mathrm{Ru}(\mathrm{bpy})_{3-n}\left(\mathrm{Me}_{2} \mathrm{Pqn}\right)_{n}\right]\left(\mathrm{PF}_{6}\right)_{2} \quad$ (bpy $=2,2^{\prime}$-bipyridine; HUTRIV, HUTPCB, HUTPUH and HUTQAO; Suzuki et al., $2003)$, and $\left[\mathrm{Pt}(\mathrm{ppy})\left(\mathrm{Me}_{2} \mathrm{Pqn}\right)\right] \mathrm{BF}_{4}$ (ppy $=2$-(2'-pyridyl)phenyl; Mori \& Suzuki, 2020). Some of the related bis(8-quinolylphosphanes) complexes are: $\left[\mathrm{Ni}\left(\mathrm{Ph}_{2} \mathrm{Pqn}\right)_{2}\right]\left(\mathrm{BF}_{4}\right)_{n}(n=1$ or 2 ; BUGDAJ, BUGDEN and BUGDOX) and $\left[\mathrm{Ni}(\mathrm{MePhPqn})_{2}\right]\left(\mathrm{BF}_{4}\right)_{2}$ (BUGDIR; Hashimoto et al., 2010), $\left[\mathrm{Pd}\left(\mathrm{Ph}_{2} \mathrm{Pqn}\right)_{2}\right] X_{2} \quad\left(X_{2}=\mathrm{Cl}_{2}, \mathrm{Br}_{2}\right.$ or $\mathrm{ClBF}_{4} ;$ FERZOS, FERZUY and FESBAH; Suzuki, 2004), $\left[\mathrm{Cu}\left(\mathrm{Ph}_{2} \mathrm{Pqn}\right)_{2}\right] \mathrm{BF}_{4}$ (OZILEP and OZILEP01; Suzuki et al., 2011) and $\left[\mathrm{Cu}\left(\mathrm{Ph}_{2} \mathrm{Pqn}\right)_{2}\right] \mathrm{PF}_{6}$ (NOPNIQ; Tsukuda et al., 2009).

\section{Synthesis and crystallization}

The ligand, $\mathrm{Me}_{2} \mathrm{Pqn}$, and the nickel(II) complexes, $\left[\mathrm{Ni}\left(\mathrm{Me}_{2} \mathrm{Pqn}\right)_{2}\right]\left(\mathrm{ClO}_{4}\right)_{2}$, were prepared according to the method reported previously (Suzuki et al., 1995). Single crystals of 1 suitable for an X-ray diffraction study were obtained by recrystallization from nitromethane by diffusion of diisopropyl ether. The platinum(II) complex, $\left[\mathrm{Pt}\left(\mathrm{Me}_{2} \mathrm{Pqn}\right)_{2}\right]\left(\mathrm{BF}_{4}\right)_{2}$, was prepared by the following method. A methanol $(5 \mathrm{ml})$ solution of $\mathrm{Me}_{2} \mathrm{Pqn}(0.76 \mathrm{mmol})$ was added dropwise with stirring to a dichloromethane solution $(10 \mathrm{ml})$ of $\left[\mathrm{PtCl}_{2}(\mathrm{EtCN})_{2}\right](0.105 \mathrm{~g}, 0.278 \mathrm{mmol})$, and the mixture was stirred for $24 \mathrm{~h}$ at room temperature. After removal of the resulting precipitate, the filtrate was concentrated to $c a 5 \mathrm{ml}$ using a rotary evaporator. A large excess amount of a methanol solution of $\mathrm{NaBF}_{4}$ was added, and the mixture was stirred for $1 \mathrm{~h}$ at room temperature. The resulting pale-yellow precipitate was collected by filtration, washed with water $(5 \mathrm{ml})$ and diethyl ether $(10 \mathrm{ml})$, and dried in vacuo. Colorless platelet-shaped crystals of $\left[\mathrm{Pt}\left(\mathrm{Me}_{2} \mathrm{Pqn}\right)_{2}\right]\left(\mathrm{BF}_{4}\right)_{2} \cdot \mathrm{CH}_{3} \mathrm{CN}$ (2) were obtained by recrystallization from an acetonitrile solution by diffusion of diisopropyl ether. Yield: $0.126 \mathrm{~g}(61 \%)$. Analysis calculated for $\mathrm{C}_{24} \mathrm{H}_{27} \mathrm{~B}_{2} \mathrm{~F}_{8} \mathrm{~N}_{3} \mathrm{P}_{2} \mathrm{Pt}$ : C, 35.37; H, 3.24; $\mathrm{N}$, $3.75 \%$. Found (after completely drying): C, 35.39; H, 2.89; N, $3.74 \%$.

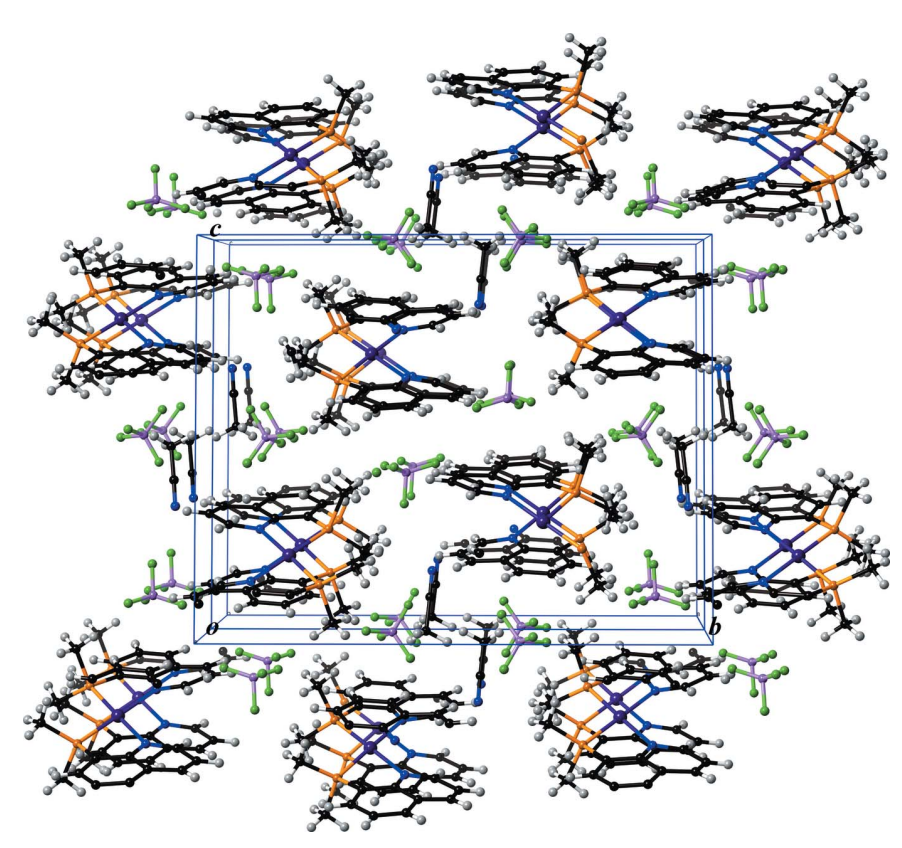

Figure 6

A packing drawing of $\left[\mathrm{Pt}\left(\mathrm{Me}_{2} \mathrm{Pqn}\right)_{2}\right]\left(\mathrm{BF}_{4}\right)_{2} \cdot \mathrm{CH}_{3} \mathrm{CN}$ (2) along the crystallographic $a$ axis (two unit cells are shown). Color code: Pt, purple; $\mathrm{P}$, orange; F, green; $\mathrm{N}$, blue; $\mathrm{C}$, black; $\mathrm{B}$, pale purple and $\mathrm{H}$, gray.

\section{Refinement}

Crystal data, data collection and structure refinement details are summarized in Table 3. All $\mathrm{H}$ atoms were refined using a riding model, with $\mathrm{C}-\mathrm{H}=0.95$ (aromatic) or 0.98 (methyl) $\AA$ and $U_{\text {iso }}(\mathrm{H})=1.2 U_{\text {eq }}(\mathrm{C})$. In the analysis of $\mathbf{2}$, two sets of $\mathrm{F}$ atoms for one of the two $\mathrm{BF}_{4}{ }^{-}$anions were introduced as positionally disordered atoms, and their occupation parameters were refined with suitable restrictions [the final major:minor occupancy ratio was 0.573 (10):0.427 (10)].

\section{Funding information}

This work was partly supported by JSPS KAKENHI Grant No. 18 K05146.

\section{References}

Burla, M. C., Caliandro, R., Camalli, M., Carrozzini, B., Cascarano, G. L., Giacovazzo, C., Mallamo, M., Mazzone, A., Polidori, G. \& Spagna, R. (2012). J. Appl. Cryst. 45, 357-361.

Cai, T., Yang, Y., Li, W.-W., Qin, W.-B. \& Wen, T.-B. (2018). Chem. Eur. J. 24, 1606-1618.

CrystalMaker Software (2017). CrystalMaker. CrystalMaker Software, Bicester, Oxfordshire, England.

Groom, C. R., Bruno, I. J., Lightfoot, M. P. \& Ward, S. C. (2016). Acta Cryst. B72, 171-179.

Hashimoto, A., Yamaguchi, H., Suzuki, T., Kashiwabara, K., Kojima, M. \& Takagi, H. D. (2010). Eur. J. Inorg. Chem. pp. 39-47.

Hopkins, J. A., Lionetti, D., Day, V. W. \& Blakemore, J. D. (2019). Organometallics, 38, 1300-1310.

Mori, M., Sunatsuki, Y. \& Suzuki, T. (2020). Manuscript to be submitted: CCDC No. 2027242-2027255.

Mori, M. \& Suzuki, T. (2020). Inorg. Chim. Acta, 512, 119862.

Rigaku (1995). ABSCOR. Rigaku Corporation, Tokyo, Japan.

Rigaku (1998). PROCESS-AUTO. Rigaku Corporation, Tokyo, Japan. 
Table 3

Experimental details.

1

Crystal data

Chemical formula

$M_{\mathrm{r}}$

Crystal system, space group

Temperature $(\mathrm{K})$

$a, b, c(\AA)$

$\beta\left({ }^{\circ}\right)$

$V\left(\AA^{3}\right)$

$Z$

Radiation type

$\mu\left(\mathrm{mm}^{-1}\right)$

Crystal size (mm)

Data collection

Diffractometer

Absorption correction

$T_{\min }, T_{\max }$

No. of measured, independent and

observed $[I>2 \sigma(I)]$ reflections

$R_{\text {int }}$

$(\sin \theta / \lambda)_{\max }\left(\AA^{-1}\right)$

Refinemen

$R\left[F^{2}>2 \sigma\left(F^{2}\right)\right], w R\left(F^{2}\right), S$

No. of reflections

No. of parameters

$\mathrm{H}$-atom treatment

$\Delta \rho_{\max }, \Delta \rho_{\min }\left(\mathrm{e} \AA^{-3}\right)$

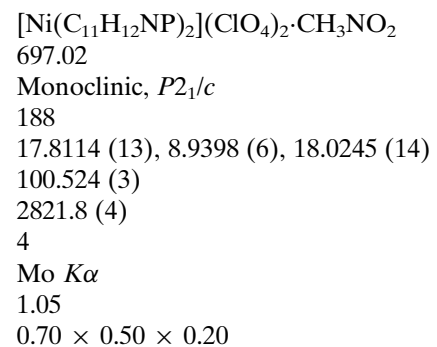

Rigaku R-AXIS RAPID

Multi-scan (ABSCOR; Rigaku, 1995)

$0.379,0.811$

26671, 6459, 5440

0.041

0.649

$0.043,0.117,1.05$

6459

370

$\mathrm{H}$-atom parameters constrained

$1.03,-0.49$
2

$\left[\mathrm{Pt}\left(\mathrm{C}_{11} \mathrm{H}_{12} \mathrm{NP}\right)_{2}\right]\left(\mathrm{BF}_{4}\right)_{2} \cdot \mathrm{C}_{2} \mathrm{H}_{3} \mathrm{~N}$

788.13

Monoclinic, $P 2_{1} / n$

188

7.9102 (3), 21.0833 (5), 16.7519 (4)

$95.3931(11)$

2781.42 (15)

4

Mo $K \alpha$

5.23

$0.30 \times 0.30 \times 0.10$

Rigaku R-AXIS RAPID

Numerical (NUMABS; Rigaku, 1999)

$0.338,0.594$

$43967,6350,5435$

0.042

0.649

$0.033,0.084,1.07$

6350

366

$\mathrm{H}$-atom parameters constrained

$1.42,-1.45$

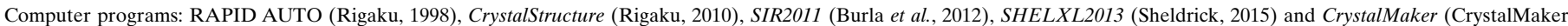
Software, 2017).

Rigaku (1999). NUMABS. Rigaku Corporation, Tokyo, Japan.

Rigaku (2010). CrystalStructure. Rigaku Corporation, Tokyo, Japan.

Salem, G. \& Wild, S. B. (1992). Inorg. Chem. 31, 581-586.

Scattolin, T., Visentin, F., Santo, C., Bertolasi, V. \& Canovese, L. (2017). Dalton Trans. 46, 5210-5217.

Sheldrick, G. M. (2015). Acta Cryst. C71, 3-8.

Suzuki, T. (2004). Bull. Chem. Soc. Jpn, 77, 1869-1876.

Suzuki, T., Kashiwabara, K. \& Fujita, J. (1995). Bull. Chem. Soc. Jpn, 68, 1619-1626.
Suzuki, T., Kuchiyama, T., Kishi, S., Kaizaki, S., Takagi, H.-D. \& Kato, M. (2003). Inorg. Chem. 42, 785-795.

Suzuki, T., Yamaguchi, H., Hashimoto, A., Nozaki, K., Doi, M., Inazumi, N., Ikeda, N., Kawata, S., Kojima, M. \& Takagi, H. D. (2011). Inorg. Chem. 50, 3981-3987.

Tsukuda, T., Nishigata, C., Arai, K. \& Tsubomura, T. (2009). Polyhedron, 28, 7-12.

Yang, L., Powell, D. R. \& Houser, R. P. (2007). Dalton Trans. pp. 955964. 


\section{supporting information}

Acta Cryst. (2020). E76, 1813-1817 [https://doi.org/10.1107/S2056989020014437]

\section{Comparison of molecular structures of cis-bis[8-(dimethyl-} phosphanyl)quinoline]nickel(II) and -platinum(II) complex cations

\section{Masatoshi Mori and Takayoshi Suzuki}

Computing details

For both structures, data collection: RAPID AUTO (Rigaku, 1998); cell refinement: RAPID AUTO (Rigaku, 1998); data reduction: CrystalStructure (Rigaku, 2010); program(s) used to solve structure: SIR2011 (Burla et al., 2012); program(s) used to refine structure: SHELXL2013 (Sheldrick, 2015); molecular graphics: CrystalMaker (CrystalMaker Software, 2017); software used to prepare material for publication: SHELXL2013 (Sheldrick, 2015).

(SP-4-2)-cis-Bis[8-(dimethylphosphanyl)quinoline- $\left.\kappa^{2} N, P\right]$ nickel(II) bis(perchlorate) nitromethane monosolvate

Crystal data

$\left[\mathrm{Ni}\left(\mathrm{C}_{11} \mathrm{H}_{12} \mathrm{NP}\right)_{2}\right]\left(\mathrm{ClO}_{4}\right)_{2} \cdot \mathrm{CH}_{3} \mathrm{NO}_{2}$

$M_{r}=697.02$

Monoclinic, $P 2{ }_{1} / c$

$a=17.8114(13) \AA$

$b=8.9398(6) \AA$

$c=18.0245(14) \AA$

$\beta=100.524(3)^{\circ}$

$V=2821.8(4) \AA^{3}$

$Z=4$

\section{Data collection}

Rigaku R-AXIS RAPID diffractometer

Radiation source: fine-focus sealed tube Detector resolution: 10.000 pixels $\mathrm{mm}^{-1}$ $\omega$ scans

Absorption correction: multi-scan

(ABSCOR; Rigaku, 1995)

$T_{\min }=0.379, T_{\max }=0.811$

\section{Refinement}

Refinement on $F^{2}$

Least-squares matrix: full

$R\left[F^{2}>2 \sigma\left(F^{2}\right)\right]=0.043$

$w R\left(F^{2}\right)=0.117$

$S=1.05$

6459 reflections

370 parameters

0 restraints
$F(000)=1432$

$D_{\mathrm{x}}=1.641 \mathrm{Mg} \mathrm{m}^{-3}$

Mo $K \alpha$ radiation, $\lambda=0.71075 \AA$

Cell parameters from 20058 reflections

$\theta=3.2-27.6^{\circ}$

$\mu=1.05 \mathrm{~mm}^{-1}$

$T=188 \mathrm{~K}$

Block, red

$0.70 \times 0.50 \times 0.20 \mathrm{~mm}$

26671 measured reflections

6459 independent reflections

5440 reflections with $I>2 \sigma(I)$

$R_{\text {int }}=0.041$

$\theta_{\max }=27.5^{\circ}, \theta_{\min }=3.2^{\circ}$

$h=-23 \rightarrow 23$

$k=-11 \rightarrow 10$

$l=-23 \rightarrow 23$

Primary atom site location: structure-invariant direct methods

Secondary atom site location: difference Fourier map

Hydrogen site location: inferred from neighbouring sites

$\mathrm{H}$-atom parameters constrained 
$w=1 /\left[\sigma^{2}\left(F_{\mathrm{o}}^{2}\right)+(0.0596 P)^{2}+2.7492 P\right]$

where $P=\left(F_{\mathrm{o}}^{2}+2 F_{\mathrm{c}}^{2}\right) / 3$

$(\Delta / \sigma)_{\max }=0.001$

$$
\Delta \rho_{\max }=1.03 \mathrm{e} \AA^{-3}
$$$$
\Delta \rho_{\text {min }}=-0.49 \text { e } \AA^{-3}
$$

\section{Special details}

Geometry. All esds (except the esd in the dihedral angle between two 1.s. planes) are estimated using the full covariance matrix. The cell esds are taken into account individually in the estimation of esds in distances, angles and torsion angles; correlations between esds in cell parameters are only used when they are defined by crystal symmetry. An approximate (isotropic) treatment of cell esds is used for estimating esds involving l.s. planes.

Fractional atomic coordinates and isotropic or equivalent isotropic displacement parameters $\left(\AA^{2}\right)$

\begin{tabular}{|c|c|c|c|c|}
\hline & $x$ & $y$ & $z$ & $U_{\text {iso }} * / U_{\text {eq }}$ \\
\hline Ni1 & $0.74556(2)$ & $0.21692(3)$ & $0.05020(2)$ & $0.02369(10)$ \\
\hline $\mathrm{Cl1}$ & $0.60962(3)$ & $0.03126(7)$ & $-0.11398(3)$ & $0.02893(15)$ \\
\hline $\mathrm{Cl} 2$ & $0.90376(4)$ & $0.47701(8)$ & 0.15809 (4) & $0.03678(16)$ \\
\hline $\mathrm{P} 1$ & $0.79934(4)$ & $0.00551(7)$ & $0.08449(4)$ & $0.02582(15)$ \\
\hline $\mathrm{P} 2$ & $0.68019(4)$ & $0.22150(7)$ & $0.13957(3)$ & $0.02357(14)$ \\
\hline $\mathrm{O} 1$ & $0.57753(13)$ & $0.1576(2)$ & $-0.15803(13)$ & $0.0477(5)$ \\
\hline $\mathrm{O} 2$ & $0.59943(13)$ & $-0.1010(2)$ & -0.15961 & $0.0409(5)$ \\
\hline $\mathrm{O} 3$ & $0.57362(13)$ & $0.0136(3)$ & $-0.04995(12)$ & $0.0495(6)$ \\
\hline $\mathrm{O} 4$ & $0.68979(11)$ & $0.0582(3)$ & $-0.08854(13)$ & $0.0475(5)$ \\
\hline $\mathrm{O} 5$ & $0.94703(18)$ & $0.4498(6)$ & $0.1033(2)$ & $0.1249(18)$ \\
\hline O6 & $0.9264(2)$ & $0.6110(4)$ & $0.1969(2)$ & $0.1026(13)$ \\
\hline $\mathrm{O} 7$ & $0.9117(3)$ & $0.3654(5)$ & $0.2115(3)$ & $0.156(2)$ \\
\hline O8 & $0.82342(15)$ & $0.4791(4)$ & $0.12503(17)$ & $0.0726(8)$ \\
\hline O9 & $0.77720(18)$ & $0.6729(4)$ & $-0.1337(2)$ & $0.1098(15)$ \\
\hline $\mathrm{O} 10$ & $0.70736(19)$ & $0.6516(3)$ & $-0.24168(17)$ & $0.0800(10)$ \\
\hline N1 & $0.82335(12)$ & $0.2389(2)$ & $-0.01392(12)$ & $0.0267(4)$ \\
\hline $\mathrm{N} 2$ & $0.67687(12)$ & $0.3788(2)$ & $0.00323(11)$ & $0.0251(4)$ \\
\hline N3 & $0.75088(15)$ & 0.7214 & $-0.19493(17)$ & $0.0435(6)$ \\
\hline $\mathrm{C} 1$ & $0.83949(15)$ & 0.3680 & $-0.04390(15)$ & $0.0334(6)$ \\
\hline H1 & 0.8160 & 0.4564 & -0.0300 & $0.040 *$ \\
\hline $\mathrm{C} 2$ & $0.88960(16)$ & $0.3797(4)$ & $-0.09511(16)$ & $0.0408(7)$ \\
\hline $\mathrm{H} 2$ & 0.9004 & 0.4747 & -0.1144 & $0.049 *$ \\
\hline $\mathrm{C} 3$ & $0.92279(16)$ & $0.2540(4)$ & $-0.11705(16)$ & $0.0408(7)$ \\
\hline $\mathrm{H} 3$ & 0.9538 & 0.2594 & -0.1546 & $0.049 *$ \\
\hline $\mathrm{C} 4$ & $0.91060(14)$ & $0.1157(4)$ & $-0.08344(15)$ & $0.0349(6)$ \\
\hline $\mathrm{C} 5$ & $0.94656(15)$ & -0.0205 (4) & $-0.09839(17)$ & $0.0413(7)$ \\
\hline H5 & 0.9771 & -0.0228 & -0.1364 & $0.050 *$ \\
\hline C6 & $0.93793(16)$ & $-0.1476(4)$ & -0.05907 (18) & $0.0429(7)$ \\
\hline H6 & 0.9621 & -0.2375 & -0.0702 & $0.051 *$ \\
\hline $\mathrm{C} 7$ & $0.89332(15)$ & -0.1466 & -0.00189 (17) & $0.0364(6)$ \\
\hline H7 & 0.8894 & -0.2343 & 0.0270 & $0.044^{*}$ \\
\hline $\mathrm{C} 8$ & $0.85549(14)$ & $-0.0183(3)$ & $0.01185(15)$ & $0.0286(5)$ \\
\hline C9 & $0.86259(13)$ & $0.1132(3)$ & $-0.02987(14)$ & $0.0277(5)$ \\
\hline $\mathrm{C} 10$ & $0.86537(16)$ & $-0.0119(3)$ & $0.17317(16)$ & $0.0370(6)$ \\
\hline H10A & 0.9035 & 0.0681 & 0.1772 & $0.044^{*}$ \\
\hline H10B & 0.8910 & -0.1092 & 0.1753 & $0.044 *$ \\
\hline
\end{tabular}




$\begin{array}{lllll}\text { H10C } & 0.8373 & -0.0040 & 0.2150 & 0.044^{*} \\ \text { C11 } & 0.73838(16) & -0.1577(3) & 0.07758(19) & 0.0391(6) \\ \text { H11A } & 0.7694 & -0.2480 & 0.0768 & 0.047^{*} \\ \text { H11B } & 0.7002 & -0.1523 & 0.0311 & 0.047^{*} \\ \text { H11C } & 0.7126 & -0.1611 & 0.1212 & 0.047^{*} \\ \text { C12 } & 0.66794(15) & 0.4179(3) & -0.06857(14) & 0.0298(5) \\ \text { H12 } & 0.6936 & 0.3612 & -0.1009 & 0.036^{*} \\ \text { C13 } & 0.62244(16) & 0.5391(3) & -0.09962(16) & 0.0354(6) \\ \text { H13 } & 0.6166 & 0.5605 & -0.1520 & 0.042^{*} \\ \text { C14 } & 0.58701(15) & 0.6251(3) & -0.05472(16) & 0.0349(6) \\ \text { H14 } & 0.5590 & 0.7111 & -0.0745 & 0.042^{*} \\ \text { C15 } & 0.59216(14) & 0.5853(3) & 0.02189(15) & 0.0287(5) \\ \text { C16 } & 0.55524(15) & 0.6643(3) & 0.07288(17) & 0.0343(6) \\ \text { H16 } & 0.5277 & 0.7531 & 0.0569 & 0.041^{*} \\ \text { C17 } & 0.55876(15) & 0.6142(3) & 0.14475(17) & 0.0357(6) \\ \text { H17 } & 0.5353 & 0.6702 & 0.1791 & 0.043^{*} \\ \text { C18 } & 0.59706(15) & 0.4793(3) & 0.16850(16) & 0.0320(6) \\ \text { H18 } & 0.5962 & 0.4416 & 0.2176 & 0.038^{*} \\ \text { C19 } & 0.63542(14) & 0.4026(3) & 0.12141(14) & 0.0262(5) \\ \text { C20 } & 0.63564(14) & 0.4568(3) & 0.04816(14) & 0.0250(5) \\ \text { C21 } & 0.60140(15) & 0.0941(3) & 0.13956(17) & 0.0348(6) \\ \text { H21A } & 0.5720 & 0.0851 & 0.0882 & 0.042^{*} \\ \text { H21B } & 0.5683 & 0.1325 & 0.1731 & 0.042^{*} \\ \text { H21C } & 0.6212 & -0.0044 & 0.1573 & 0.042^{*} \\ \text { C22 } & 0.73083(16) & 0.2268(3) & 0.23602(14) & 0.0332(6) \\ \text { H22A } & 0.6946 & 0.2457 & 0.2698 & 0.040^{*} \\ \text { H22B } & 0.7690 & 0.3069 & 0.2416 & 0.040^{*} \\ \text { H22C } & 0.7563 & 0.1306 & 0.2489 & 0.040^{*} \\ \text { C23 } & 0.77162(18) & 0.8734(3) & -0.21331(18) & 0.0415(7) \\ \text { H23A } & 0.7415 & 0.9028 & -0.2622 & 0.050^{*} \\ \text { H23B } & 0.8261 & 0.8769 & -0.2158 & 0.050^{*} \\ \text { H23C } & 0.7611 & 0.9425 & -0.1742 & 0.050^{*}\end{array}$

Atomic displacement parameters $\left(\AA^{2}\right)$

\begin{tabular}{lllllll}
\hline & $U^{11}$ & $U^{22}$ & $U^{33}$ & $U^{12}$ & $U^{13}$ & $U^{23}$ \\
\hline Ni1 & $0.02478(17)$ & $0.02146(17)$ & $0.02684(18)$ & $0.00232(12)$ & $0.00998(12)$ & $0.00229(11)$ \\
C11 & $0.0286(3)$ & $0.0280(3)$ & $0.0311(3)$ & $0.0003(2)$ & $0.0078(2)$ & $-0.0003(2)$ \\
C12 & $0.0402(4)$ & $0.0324(3)$ & $0.0390(4)$ & $-0.0014(3)$ & $0.0107(3)$ & $-0.0005(3)$ \\
P1 & $0.0232(3)$ & $0.0227(3)$ & $0.0327(3)$ & $0.0020(2)$ & $0.0082(2)$ & $0.0015(2)$ \\
P2 & $0.0240(3)$ & $0.0233(3)$ & $0.0246(3)$ & $0.0029(2)$ & $0.0078(2)$ & $0.0016(2)$ \\
O1 & $0.0566(13)$ & $0.0350(11)$ & $0.0505(13)$ & $0.0162(10)$ & $0.0072(11)$ & $0.0061(9)$ \\
O2 & $0.0594(13)$ & $0.0287(10)$ & $0.0360(11)$ & $-0.0060(9)$ & $0.0123(10)$ & $-0.0034(8)$ \\
O3 & $0.0531(13)$ & $0.0633(15)$ & $0.0376(12)$ & $-0.0076(11)$ & $0.0227(10)$ & $-0.0053(10)$ \\
O4 & $0.0267(10)$ & $0.0517(13)$ & $0.0637(14)$ & $-0.0025(9)$ & $0.0070(9)$ & $-0.0069(11)$ \\
O5 & $0.0573(18)$ & $0.220(5)$ & $0.107(3)$ & $-0.033(2)$ & $0.0413(19)$ & $-0.094(3)$ \\
O6 & $0.091(2)$ & $0.082(2)$ & $0.149(3)$ & $-0.041(2)$ & $0.059(2)$ & $-0.062(2)$ \\
O7 & $0.164(4)$ & $0.120(4)$ & $0.159(4)$ & $-0.038(3)$ & $-0.041(3)$ & $0.091(3)$
\end{tabular}




\begin{tabular}{|c|c|c|c|c|c|c|}
\hline O8 & $0.0430(14)$ & $0.095(2)$ & $0.080(2)$ & $0.0072(14)$ & $0.0111(13)$ & $-0.0257(16)$ \\
\hline O9 & $0.070(2)$ & $0.103(3)$ & $0.139(3)$ & $-0.0263(18)$ & $-0.029(2)$ & $0.086(2)$ \\
\hline $\mathrm{O} 10$ & $0.110(2)$ & $0.0643(17)$ & 0.0767 (19) & $-0.0462(18)$ & $0.0471(18)$ & $-0.0385(15)$ \\
\hline N1 & $0.0243(10)$ & $0.0289(10)$ & $0.0280(11)$ & $-0.0045(9)$ & $0.0071(8)$ & $-0.0013(8)$ \\
\hline $\mathrm{N} 2$ & $0.0278(10)$ & $0.0213(10)$ & $0.0271(10)$ & $-0.0013(8)$ & $0.0074(8)$ & $0.0013(8)$ \\
\hline N3 & $0.0373(13)$ & $0.0317(13)$ & $0.0643(18)$ & $0.0005(11)$ & $0.0165(13)$ & $0.0012(12)$ \\
\hline $\mathrm{C} 1$ & $0.0315(13)$ & $0.0354(14)$ & $0.0337(14)$ & $-0.0072(11)$ & $0.0073(11)$ & $0.0006(11)$ \\
\hline $\mathrm{C} 2$ & $0.0332(14)$ & $0.0522(18)$ & $0.0378(15)$ & $-0.0148(13)$ & $0.0088(12)$ & $0.0072(13)$ \\
\hline $\mathrm{C} 3$ & $0.0261(13)$ & $0.066(2)$ & $0.0326(14)$ & $-0.0110(14)$ & $0.0113(11)$ & $-0.0016(14)$ \\
\hline $\mathrm{C} 4$ & $0.0211(11)$ & $0.0549(18)$ & $0.0286(13)$ & $-0.0042(12)$ & $0.0043(10)$ & $-0.0086(12)$ \\
\hline $\mathrm{C} 5$ & $0.0213(12)$ & $0.066(2)$ & $0.0377(15)$ & $0.0004(13)$ & $0.0072(11)$ & $-0.0178(14)$ \\
\hline C6 & $0.0263(13)$ & $0.0550(19)$ & 0.0467 (17) & $0.0075(13)$ & $0.0051(12)$ & $-0.0227(15)$ \\
\hline $\mathrm{C} 7$ & $0.0275(13)$ & $0.0372(15)$ & $0.0432(16)$ & $0.0036(12)$ & $0.0026(11)$ & $-0.0096(12)$ \\
\hline $\mathrm{C} 8$ & $0.0204(11)$ & $0.0324(13)$ & $0.0328(13)$ & $-0.0007(10)$ & $0.0044(10)$ & $-0.0071(10)$ \\
\hline C9 & $0.0205(11)$ & $0.0343(13)$ & $0.0282(12)$ & $-0.0036(10)$ & $0.0038(9)$ & $-0.0080(10)$ \\
\hline C10 & $0.0324(14)$ & $0.0433(16)$ & $0.0354(15)$ & $0.0106(12)$ & $0.0069(11)$ & $0.0035(12)$ \\
\hline $\mathrm{C} 11$ & $0.0338(14)$ & $0.0282(13)$ & $0.0570(18)$ & $-0.0022(12)$ & $0.0128(13)$ & $0.0031(13)$ \\
\hline $\mathrm{C} 12$ & $0.0320(13)$ & $0.0285(12)$ & $0.0295(13)$ & $-0.0043(11)$ & $0.0068(10)$ & $0.0019(10)$ \\
\hline $\mathrm{C} 13$ & $0.0365(14)$ & $0.0350(14)$ & $0.0332(14)$ & $-0.0058(12)$ & $0.0023(11)$ & $0.0129(11)$ \\
\hline C14 & $0.0321(13)$ & $0.0256(13)$ & $0.0450(16)$ & $-0.0024(11)$ & $0.0014(12)$ & $0.0106(11)$ \\
\hline $\mathrm{C} 15$ & $0.0244(11)$ & $0.0212(11)$ & $0.0394(14)$ & $-0.0027(10)$ & $0.0029(10)$ & $0.0006(10)$ \\
\hline $\mathrm{C} 16$ & $0.0273(12)$ & $0.0226(12)$ & $0.0517(17)$ & $0.0033(10)$ & $0.0035(12)$ & $-0.0029(11)$ \\
\hline $\mathrm{C} 17$ & $0.0292(13)$ & $0.0324(14)$ & $0.0457(16)$ & $0.0073(11)$ & $0.0075(11)$ & $-0.0120(12)$ \\
\hline $\mathrm{C} 18$ & $0.0288(12)$ & $0.0345(14)$ & $0.0330(14)$ & $0.0043(11)$ & $0.0065(10)$ & $-0.0053(11)$ \\
\hline C19 & $0.0247(11)$ & $0.0256(12)$ & $0.0279(12)$ & $0.0022(10)$ & $0.0039(9)$ & $-0.0016(9)$ \\
\hline $\mathrm{C} 20$ & $0.0239(11)$ & $0.0199(11)$ & $0.0309(12)$ & $-0.0014(9)$ & $0.0043(9)$ & $-0.0015(9)$ \\
\hline $\mathrm{C} 21$ & $0.0294(13)$ & $0.0337(14)$ & $0.0436(15)$ & $-0.0020(11)$ & $0.0128(11)$ & $0.0019(12)$ \\
\hline $\mathrm{C} 22$ & $0.0383(14)$ & $0.0352(14)$ & $0.0256(13)$ & $0.0099(12)$ & $0.0047(11)$ & $0.0011(10)$ \\
\hline $\mathrm{C} 23$ & $0.0407(15)$ & $0.0336(15)$ & $0.0501(17)$ & $-0.0029(13)$ & $0.0081(13)$ & $0.0051(13)$ \\
\hline
\end{tabular}

Geometric parameters $\left(\AA,{ }^{o}\right)$

\begin{tabular}{llll}
\hline $\mathrm{Ni} 1-\mathrm{N} 1$ & $1.970(2)$ & $\mathrm{C} 6-\mathrm{C} 7$ & $1.412(4)$ \\
$\mathrm{N} i 1-\mathrm{N} 2$ & $1.982(2)$ & $\mathrm{C} 6-\mathrm{H} 6$ & 0.9500 \\
$\mathrm{~N} 11-\mathrm{P} 2$ & $2.1534(7)$ & $\mathrm{C} 7-\mathrm{C} 8$ & $1.376(4)$ \\
$\mathrm{N} 11-\mathrm{P} 1$ & $2.1576(7)$ & $\mathrm{C} 7-\mathrm{H} 7$ & 0.9500 \\
$\mathrm{C} 11-\mathrm{O} 3$ & $1.428(2)$ & $\mathrm{C} 8-\mathrm{C} 9$ & $1.413(4)$ \\
$\mathrm{C} 1-\mathrm{O} 2$ & $1.433(2)$ & $\mathrm{C} 10-\mathrm{H} 10 \mathrm{~A}$ & 0.9800 \\
$\mathrm{C} 11-\mathrm{O} 1$ & $1.438(2)$ & $\mathrm{C} 10-\mathrm{H} 10 \mathrm{~B}$ & 0.9800 \\
$\mathrm{C} 11-\mathrm{O} 4$ & $1.438(2)$ & $\mathrm{C} 10-\mathrm{H} 10 \mathrm{C}$ & 0.9800 \\
$\mathrm{C} 12-\mathrm{O} 7$ & $1.376(4)$ & $\mathrm{C} 11-\mathrm{H} 11 \mathrm{~A}$ & 0.9800 \\
$\mathrm{Cl} 2-\mathrm{O} 5$ & $1.381(3)$ & $\mathrm{C} 11-\mathrm{H} 11 \mathrm{~B}$ & 0.9800 \\
$\mathrm{C} 12-\mathrm{O} 6$ & $1.408(3)$ & $\mathrm{C} 11-\mathrm{H} 11 \mathrm{C}$ & 0.9800 \\
$\mathrm{Cl} 2-\mathrm{O} 8$ & $1.446(3)$ & $\mathrm{C} 12-\mathrm{C} 13$ & $1.406(4)$ \\
$\mathrm{P} 1-\mathrm{C} 8$ & $1.800(3)$ & $\mathrm{C} 12-\mathrm{H} 12$ & 0.9500 \\
$\mathrm{P} 1-\mathrm{C} 11$ & $1.809(3)$ & $\mathrm{C} 13-\mathrm{C} 14$ & $1.353(4)$ \\
$\mathrm{P} 1-\mathrm{C} 10$ & $1.810(3)$ & $\mathrm{C} 13-\mathrm{H} 13$ & 0.9500 \\
$\mathrm{P} 2-\mathrm{C} 22$ & $1.807(3)$ & $\mathrm{C} 14-\mathrm{C} 15$ & $1.413(4)$
\end{tabular}




\begin{tabular}{|c|c|c|c|}
\hline $\mathrm{P} 2-\mathrm{C} 21$ & $1.807(3)$ & $\mathrm{C} 14-\mathrm{H} 14$ & 0.9500 \\
\hline $\mathrm{P} 2-\mathrm{C} 19$ & $1.808(3)$ & $\mathrm{C} 15-\mathrm{C} 16$ & $1.413(4)$ \\
\hline $\mathrm{O} 9-\mathrm{N} 3$ & $1.199(4)$ & $\mathrm{C} 15-\mathrm{C} 20$ & $1.417(3)$ \\
\hline $\mathrm{O} 10-\mathrm{N} 3$ & $1.209(4)$ & $\mathrm{C} 16-\mathrm{C} 17$ & $1.361(4)$ \\
\hline $\mathrm{N} 1-\mathrm{C} 1$ & $1.327(3)$ & $\mathrm{C} 16-\mathrm{H} 16$ & 0.9500 \\
\hline $\mathrm{N} 1-\mathrm{C} 9$ & $1.381(3)$ & $\mathrm{C} 17-\mathrm{C} 18$ & $1.413(4)$ \\
\hline $\mathrm{N} 2-\mathrm{C} 12$ & $1.322(3)$ & $\mathrm{C} 17-\mathrm{H} 17$ & 0.9500 \\
\hline $\mathrm{N} 2-\mathrm{C} 20$ & $1.378(3)$ & $\mathrm{C} 18-\mathrm{C} 19$ & $1.367(3)$ \\
\hline $\mathrm{N} 3-\mathrm{C} 23$ & $1.462(4)$ & $\mathrm{C} 18-\mathrm{H} 18$ & 0.9500 \\
\hline $\mathrm{C} 1-\mathrm{C} 2$ & $1.400(4)$ & $\mathrm{C} 19-\mathrm{C} 20$ & $1.407(3)$ \\
\hline $\mathrm{C} 1-\mathrm{H} 1$ & 0.9500 & $\mathrm{C} 21-\mathrm{H} 21 \mathrm{~A}$ & 0.9800 \\
\hline $\mathrm{C} 2-\mathrm{C} 3$ & $1.362(5)$ & $\mathrm{C} 21-\mathrm{H} 21 \mathrm{~B}$ & 0.9800 \\
\hline $\mathrm{C} 2-\mathrm{H} 2$ & 0.9500 & $\mathrm{C} 21-\mathrm{H} 21 \mathrm{C}$ & 0.9800 \\
\hline $\mathrm{C} 3-\mathrm{C} 4$ & $1.411(4)$ & $\mathrm{C} 22-\mathrm{H} 22 \mathrm{~A}$ & 0.9800 \\
\hline $\mathrm{C} 3-\mathrm{H} 3$ & 0.9500 & $\mathrm{C} 22-\mathrm{H} 22 \mathrm{~B}$ & 0.9800 \\
\hline $\mathrm{C} 4-\mathrm{C} 9$ & $1.402(3)$ & $\mathrm{C} 22-\mathrm{H} 22 \mathrm{C}$ & 0.9800 \\
\hline $\mathrm{C} 4-\mathrm{C} 5$ & $1.424(4)$ & $\mathrm{C} 23-\mathrm{H} 23 \mathrm{~A}$ & 0.9800 \\
\hline $\mathrm{C} 5-\mathrm{C} 6$ & $1.363(5)$ & $\mathrm{C} 23-\mathrm{H} 23 \mathrm{~B}$ & 0.9800 \\
\hline $\mathrm{C} 5-\mathrm{H} 5$ & 0.9500 & $\mathrm{C} 23-\mathrm{H} 23 \mathrm{C}$ & 0.9800 \\
\hline $\mathrm{N} 1-\mathrm{Ni} 1-\mathrm{N} 2$ & $97.01(9)$ & $\mathrm{C} 9-\mathrm{C} 8-\mathrm{P} 1$ & $113.84(18)$ \\
\hline $\mathrm{N} 1-\mathrm{Ni1}-\mathrm{P} 2$ & $166.27(7)$ & $\mathrm{N} 1-\mathrm{C} 9-\mathrm{C} 4$ & $121.7(2)$ \\
\hline $\mathrm{N} 2-\mathrm{Ni1}-\mathrm{P} 2$ & $85.97(6)$ & $\mathrm{N} 1-\mathrm{C} 9-\mathrm{C} 8$ & $117.9(2)$ \\
\hline $\mathrm{N} 1-\mathrm{Ni} 1-\mathrm{P} 1$ & $86.13(7)$ & $\mathrm{C} 4-\mathrm{C} 9-\mathrm{C} 8$ & $120.3(2)$ \\
\hline $\mathrm{N} 2-\mathrm{Ni} 1-\mathrm{P} 1$ & $165.68(6)$ & $\mathrm{P} 1-\mathrm{C} 10-\mathrm{H} 10 \mathrm{~A}$ & 109.5 \\
\hline $\mathrm{P} 2-\mathrm{Ni1}-\mathrm{P} 1$ & $94.29(3)$ & $\mathrm{P} 1-\mathrm{C} 10-\mathrm{H} 10 \mathrm{~B}$ & 109.5 \\
\hline $\mathrm{O} 3-\mathrm{Cl} 1-\mathrm{O} 2$ & $110.15(13)$ & $\mathrm{H} 10 \mathrm{~A}-\mathrm{C} 10-\mathrm{H} 10 \mathrm{~B}$ & 109.5 \\
\hline $\mathrm{O} 3-\mathrm{Cl1}-\mathrm{O} 1$ & $109.95(14)$ & $\mathrm{P} 1-\mathrm{C} 10-\mathrm{H} 10 \mathrm{C}$ & 109.5 \\
\hline $\mathrm{O} 2-\mathrm{Cl1}-\mathrm{O} 1$ & $109.60(13)$ & $\mathrm{H} 10 \mathrm{~A}-\mathrm{C} 10-\mathrm{H} 10 \mathrm{C}$ & 109.5 \\
\hline $\mathrm{O} 3-\mathrm{Cl} 1-\mathrm{O} 4$ & $109.09(14)$ & $\mathrm{H} 10 \mathrm{~B}-\mathrm{C} 10-\mathrm{H} 10 \mathrm{C}$ & 109.5 \\
\hline $\mathrm{O} 2-\mathrm{Cl1}-\mathrm{O} 4$ & $109.61(13)$ & $\mathrm{P} 1-\mathrm{C} 11-\mathrm{H} 11 \mathrm{~A}$ & 109.5 \\
\hline $\mathrm{O} 1-\mathrm{Cl} 1-\mathrm{O} 4$ & $108.41(14)$ & $\mathrm{P} 1-\mathrm{C} 11-\mathrm{H} 11 \mathrm{~B}$ & 109.5 \\
\hline $\mathrm{O} 7-\mathrm{Cl} 2-\mathrm{O} 5$ & $111.9(4)$ & $\mathrm{H} 11 \mathrm{~A}-\mathrm{C} 11-\mathrm{H} 11 \mathrm{~B}$ & 109.5 \\
\hline $\mathrm{O} 7-\mathrm{Cl} 2-\mathrm{O} 6$ & $106.6(3)$ & $\mathrm{P} 1-\mathrm{C} 11-\mathrm{H} 11 \mathrm{C}$ & 109.5 \\
\hline $\mathrm{O} 5-\mathrm{Cl} 2-\mathrm{O} 6$ & $111.0(2)$ & $\mathrm{H} 11 \mathrm{~A}-\mathrm{C} 11-\mathrm{H} 11 \mathrm{C}$ & 109.5 \\
\hline $\mathrm{O} 7-\mathrm{Cl} 2-\mathrm{O} 8$ & $105.4(3)$ & $\mathrm{H} 11 \mathrm{~B}-\mathrm{C} 11-\mathrm{H} 11 \mathrm{C}$ & 109.5 \\
\hline $\mathrm{O} 5-\mathrm{Cl} 2-\mathrm{O} 8$ & $110.3(2)$ & $\mathrm{N} 2-\mathrm{C} 12-\mathrm{C} 13$ & $123.4(2)$ \\
\hline $\mathrm{O} 6-\mathrm{Cl} 2-\mathrm{O} 8$ & $111.5(2)$ & $\mathrm{N} 2-\mathrm{C} 12-\mathrm{H} 12$ & 118.3 \\
\hline $\mathrm{C} 8-\mathrm{P} 1-\mathrm{C} 11$ & $105.12(13)$ & $\mathrm{C} 13-\mathrm{C} 12-\mathrm{H} 12$ & 118.3 \\
\hline $\mathrm{C} 8-\mathrm{P} 1-\mathrm{C} 10$ & $105.93(12)$ & $\mathrm{C} 14-\mathrm{C} 13-\mathrm{C} 12$ & $119.8(3)$ \\
\hline $\mathrm{C} 11-\mathrm{P} 1-\mathrm{C} 10$ & $106.05(15)$ & $\mathrm{C} 14-\mathrm{C} 13-\mathrm{H} 13$ & 120.1 \\
\hline $\mathrm{C} 8-\mathrm{P} 1-\mathrm{Ni} 1$ & $99.90(9)$ & $\mathrm{C} 12-\mathrm{C} 13-\mathrm{H} 13$ & 120.1 \\
\hline C11-P1-Ni1 & $117.20(10)$ & $\mathrm{C} 13-\mathrm{C} 14-\mathrm{C} 15$ & $119.2(2)$ \\
\hline $\mathrm{C} 10-\mathrm{P} 1-\mathrm{Ni} 1$ & $120.75(10)$ & $\mathrm{C} 13-\mathrm{C} 14-\mathrm{H} 14$ & 120.4 \\
\hline $\mathrm{C} 22-\mathrm{P} 2-\mathrm{C} 21$ & $105.52(14)$ & $\mathrm{C} 15-\mathrm{C} 14-\mathrm{H} 14$ & 120.4 \\
\hline $\mathrm{C} 22-\mathrm{P} 2-\mathrm{C} 19$ & $106.08(12)$ & $\mathrm{C} 14-\mathrm{C} 15-\mathrm{C} 16$ & $123.7(2)$ \\
\hline $\mathrm{C} 21-\mathrm{P} 2-\mathrm{C} 19$ & $104.32(12)$ & $\mathrm{C} 14-\mathrm{C} 15-\mathrm{C} 20$ & 117.7 (2) \\
\hline $\mathrm{C} 22-\mathrm{P} 2-\mathrm{Ni} 1$ & $118.50(10)$ & $\mathrm{C} 16-\mathrm{C} 15-\mathrm{C} 20$ & $118.6(2)$ \\
\hline
\end{tabular}




\begin{tabular}{|c|c|c|c|}
\hline $\mathrm{C} 21-\mathrm{P} 2-\mathrm{Ni} 1$ & $120.92(10)$ & $\mathrm{C} 17-\mathrm{C} 16-\mathrm{C} 15$ & $120.6(2)$ \\
\hline $\mathrm{C} 19-\mathrm{P} 2-\mathrm{Ni} 1$ & $99.35(8)$ & $\mathrm{C} 17-\mathrm{C} 16-\mathrm{H} 16$ & 119.7 \\
\hline $\mathrm{C} 1-\mathrm{N} 1-\mathrm{C} 9$ & $117.8(2)$ & $\mathrm{C} 15-\mathrm{C} 16-\mathrm{H} 16$ & 119.7 \\
\hline $\mathrm{C} 1-\mathrm{N} 1-\mathrm{Ni} 1$ & $123.61(18)$ & $\mathrm{C} 16-\mathrm{C} 17-\mathrm{C} 18$ & $120.3(2)$ \\
\hline $\mathrm{C} 9-\mathrm{N} 1-\mathrm{Ni} 1$ & $118.53(17)$ & $\mathrm{C} 16-\mathrm{C} 17-\mathrm{H} 17$ & 119.8 \\
\hline $\mathrm{C} 12-\mathrm{N} 2-\mathrm{C} 20$ & $117.4(2)$ & $\mathrm{C} 18-\mathrm{C} 17-\mathrm{H} 17$ & 119.8 \\
\hline $\mathrm{C} 12-\mathrm{N} 2-\mathrm{Ni} 1$ & $124.45(18)$ & $\mathrm{C} 19-\mathrm{C} 18-\mathrm{C} 17$ & $120.6(3)$ \\
\hline $\mathrm{C} 20-\mathrm{N} 2-\mathrm{Ni} 1$ & $118.18(16)$ & $\mathrm{C} 19-\mathrm{C} 18-\mathrm{H} 18$ & 119.7 \\
\hline $\mathrm{O} 9-\mathrm{N} 3-\mathrm{O} 10$ & $123.2(3)$ & $\mathrm{C} 17-\mathrm{C} 18-\mathrm{H} 18$ & 119.7 \\
\hline $\mathrm{O} 9-\mathrm{N} 3-\mathrm{C} 23$ & $118.2(3)$ & $\mathrm{C} 18-\mathrm{C} 19-\mathrm{C} 20$ & $119.8(2)$ \\
\hline $\mathrm{O} 10-\mathrm{N} 3-\mathrm{C} 23$ & $118.6(3)$ & $\mathrm{C} 18-\mathrm{C} 19-\mathrm{P} 2$ & $126.2(2)$ \\
\hline $\mathrm{N} 1-\mathrm{C} 1-\mathrm{C} 2$ & $123.0(3)$ & $\mathrm{C} 20-\mathrm{C} 19-\mathrm{P} 2$ & $113.68(18)$ \\
\hline $\mathrm{N} 1-\mathrm{C} 1-\mathrm{H} 1$ & 118.5 & $\mathrm{~N} 2-\mathrm{C} 20-\mathrm{C} 19$ & $118.0(2)$ \\
\hline $\mathrm{C} 2-\mathrm{C} 1-\mathrm{H} 1$ & 118.5 & $\mathrm{~N} 2-\mathrm{C} 20-\mathrm{C} 15$ & $122.1(2)$ \\
\hline $\mathrm{C} 3-\mathrm{C} 2-\mathrm{C} 1$ & $119.5(3)$ & $\mathrm{C} 19-\mathrm{C} 20-\mathrm{C} 15$ & $119.8(2)$ \\
\hline $\mathrm{C} 3-\mathrm{C} 2-\mathrm{H} 2$ & 120.3 & $\mathrm{P} 2-\mathrm{C} 21-\mathrm{H} 21 \mathrm{~A}$ & 109.5 \\
\hline $\mathrm{C} 1-\mathrm{C} 2-\mathrm{H} 2$ & 120.3 & $\mathrm{P} 2-\mathrm{C} 21-\mathrm{H} 21 \mathrm{~B}$ & 109.5 \\
\hline $\mathrm{C} 2-\mathrm{C} 3-\mathrm{C} 4$ & $119.3(2)$ & $\mathrm{H} 21 \mathrm{~A}-\mathrm{C} 21-\mathrm{H} 21 \mathrm{~B}$ & 109.5 \\
\hline $\mathrm{C} 2-\mathrm{C} 3-\mathrm{H} 3$ & 120.3 & $\mathrm{P} 2-\mathrm{C} 21-\mathrm{H} 21 \mathrm{C}$ & 109.5 \\
\hline $\mathrm{C} 4-\mathrm{C} 3-\mathrm{H} 3$ & 120.3 & $\mathrm{H} 21 \mathrm{~A}-\mathrm{C} 21-\mathrm{H} 21 \mathrm{C}$ & 109.5 \\
\hline $\mathrm{C} 9-\mathrm{C} 4-\mathrm{C} 3$ & $118.1(3)$ & $\mathrm{H} 21 \mathrm{~B}-\mathrm{C} 21-\mathrm{H} 21 \mathrm{C}$ & 109.5 \\
\hline $\mathrm{C} 9-\mathrm{C} 4-\mathrm{C} 5$ & $117.9(3)$ & $\mathrm{P} 2-\mathrm{C} 22-\mathrm{H} 22 \mathrm{~A}$ & 109.5 \\
\hline $\mathrm{C} 3-\mathrm{C} 4-\mathrm{C} 5$ & $123.9(3)$ & $\mathrm{P} 2-\mathrm{C} 22-\mathrm{H} 22 \mathrm{~B}$ & 109.5 \\
\hline $\mathrm{C} 6-\mathrm{C} 5-\mathrm{C} 4$ & $121.1(3)$ & $\mathrm{H} 22 \mathrm{~A}-\mathrm{C} 22-\mathrm{H} 22 \mathrm{~B}$ & 109.5 \\
\hline $\mathrm{C} 6-\mathrm{C} 5-\mathrm{H} 5$ & 119.4 & $\mathrm{P} 2-\mathrm{C} 22-\mathrm{H} 22 \mathrm{C}$ & 109.5 \\
\hline $\mathrm{C} 4-\mathrm{C} 5-\mathrm{H} 5$ & 119.4 & $\mathrm{H} 22 \mathrm{~A}-\mathrm{C} 22-\mathrm{H} 22 \mathrm{C}$ & 109.5 \\
\hline $\mathrm{C} 5-\mathrm{C} 6-\mathrm{C} 7$ & $120.4(3)$ & $\mathrm{H} 22 \mathrm{~B}-\mathrm{C} 22-\mathrm{H} 22 \mathrm{C}$ & 109.5 \\
\hline $\mathrm{C} 5-\mathrm{C} 6-\mathrm{H} 6$ & 119.8 & $\mathrm{~N} 3-\mathrm{C} 23-\mathrm{H} 23 \mathrm{~A}$ & 109.5 \\
\hline $\mathrm{C} 7-\mathrm{C} 6-\mathrm{H} 6$ & 119.8 & $\mathrm{~N} 3-\mathrm{C} 23-\mathrm{H} 23 \mathrm{~B}$ & 109.5 \\
\hline $\mathrm{C} 8-\mathrm{C} 7-\mathrm{C} 6$ & $119.7(3)$ & $\mathrm{H} 23 \mathrm{~A}-\mathrm{C} 23-\mathrm{H} 23 \mathrm{~B}$ & 109.5 \\
\hline $\mathrm{C} 8-\mathrm{C} 7-\mathrm{H} 7$ & 120.1 & $\mathrm{~N} 3-\mathrm{C} 23-\mathrm{H} 23 \mathrm{C}$ & 109.5 \\
\hline $\mathrm{C} 6-\mathrm{C} 7-\mathrm{H} 7$ & 120.1 & $\mathrm{H} 23 \mathrm{~A}-\mathrm{C} 23-\mathrm{H} 23 \mathrm{C}$ & 109.5 \\
\hline $\mathrm{C} 7-\mathrm{C} 8-\mathrm{C} 9$ & $120.2(2)$ & $\mathrm{H} 23 \mathrm{~B}-\mathrm{C} 23-\mathrm{H} 23 \mathrm{C}$ & 109.5 \\
\hline $\mathrm{C} 7-\mathrm{C} 8-\mathrm{P} 1$ & $125.8(2)$ & & \\
\hline $\mathrm{C} 9-\mathrm{N} 1-\mathrm{C} 1-\mathrm{C} 2$ & $5.1(4)$ & $\mathrm{C} 20-\mathrm{N} 2-\mathrm{C} 12-\mathrm{C} 13$ & $3.7(4)$ \\
\hline $\mathrm{Ni1}-\mathrm{N} 1-\mathrm{C} 1-\mathrm{C} 2$ & $-173.7(2)$ & $\mathrm{Ni} 1-\mathrm{N} 2-\mathrm{C} 12-\mathrm{C} 13$ & $-175.97(19)$ \\
\hline $\mathrm{N} 1-\mathrm{C} 1-\mathrm{C} 2-\mathrm{C} 3$ & $1.3(4)$ & $\mathrm{N} 2-\mathrm{C} 12-\mathrm{C} 13-\mathrm{C} 14$ & $2.1(4)$ \\
\hline $\mathrm{C} 1-\mathrm{C} 2-\mathrm{C} 3-\mathrm{C} 4$ & $-4.8(4)$ & $\mathrm{C} 12-\mathrm{C} 13-\mathrm{C} 14-\mathrm{C} 15$ & $-4.3(4)$ \\
\hline $\mathrm{C} 2-\mathrm{C} 3-\mathrm{C} 4-\mathrm{C} 9$ & $1.8(4)$ & $\mathrm{C} 13-\mathrm{C} 14-\mathrm{C} 15-\mathrm{C} 16$ & $-177.8(3)$ \\
\hline $\mathrm{C} 2-\mathrm{C} 3-\mathrm{C} 4-\mathrm{C} 5$ & $-175.6(3)$ & $\mathrm{C} 13-\mathrm{C} 14-\mathrm{C} 15-\mathrm{C} 20$ & $0.9(4)$ \\
\hline $\mathrm{C} 9-\mathrm{C} 4-\mathrm{C} 5-\mathrm{C} 6$ & $-3.4(4)$ & $\mathrm{C} 14-\mathrm{C} 15-\mathrm{C} 16-\mathrm{C} 17$ & $175.8(3)$ \\
\hline $\mathrm{C} 3-\mathrm{C} 4-\mathrm{C} 5-\mathrm{C} 6$ & $173.9(3)$ & $\mathrm{C} 20-\mathrm{C} 15-\mathrm{C} 16-\mathrm{C} 17$ & $-2.8(4)$ \\
\hline $\mathrm{C} 4-\mathrm{C} 5-\mathrm{C} 6-\mathrm{C} 7$ & $-0.5(4)$ & $\mathrm{C} 15-\mathrm{C} 16-\mathrm{C} 17-\mathrm{C} 18$ & $-2.4(4)$ \\
\hline $\mathrm{C} 5-\mathrm{C} 6-\mathrm{C} 7-\mathrm{C} 8$ & $3.0(4)$ & $\mathrm{C} 16-\mathrm{C} 17-\mathrm{C} 18-\mathrm{C} 19$ & $4.4(4)$ \\
\hline $\mathrm{C} 6-\mathrm{C} 7-\mathrm{C} 8-\mathrm{C} 9$ & $-1.5(4)$ & $\mathrm{C} 17-\mathrm{C} 18-\mathrm{C} 19-\mathrm{C} 20$ & $-1.2(4)$ \\
\hline $\mathrm{C} 6-\mathrm{C} 7-\mathrm{C} 8-\mathrm{P} 1$ & $-177.4(2)$ & $\mathrm{C} 17-\mathrm{C} 18-\mathrm{C} 19-\mathrm{P} 2$ & $-174.3(2)$ \\
\hline $\mathrm{C} 11-\mathrm{P} 1-\mathrm{C} 8-\mathrm{C} 7$ & $-47.4(3)$ & $\mathrm{C} 22-\mathrm{P} 2-\mathrm{C} 19-\mathrm{C} 18$ & $-44.2(3)$ \\
\hline
\end{tabular}




$\begin{array}{llll}\mathrm{C} 10-\mathrm{P} 1-\mathrm{C} 8-\mathrm{C} 7 & 64.6(3) & \mathrm{C} 21-\mathrm{P} 2-\mathrm{C} 19-\mathrm{C} 18 & 67.0(3) \\ \mathrm{N} 11-\mathrm{P} 1-\mathrm{C} 8-\mathrm{C} 7 & -169.2(2) & \mathrm{N} 1-\mathrm{P} 2-\mathrm{C} 19-\mathrm{C} 18 & -167.6(2) \\ \mathrm{C} 11-\mathrm{P} 1-\mathrm{C} 8-\mathrm{C} 9 & 136.4(2) & \mathrm{C} 22-\mathrm{P} 2-\mathrm{C} 19-\mathrm{C} 20 & 142.27(19) \\ \mathrm{C} 10-\mathrm{P} 1-\mathrm{C} 8-\mathrm{C} 9 & -111.6(2) & \mathrm{C} 21-\mathrm{P} 2-\mathrm{C} 19-\mathrm{C} 20 & -106.6(2) \\ \mathrm{N} 11-\mathrm{P} 1-\mathrm{C} 8-\mathrm{C} 9 & 14.54(19) & \mathrm{N} 11-\mathrm{P} 2-\mathrm{C} 19-\mathrm{C} 20 & 18.86(19) \\ \mathrm{C} 1-\mathrm{N} 1-\mathrm{C} 9-\mathrm{C} 4 & -8.2(4) & \mathrm{C} 12-\mathrm{N} 2-\mathrm{C} 20-\mathrm{C} 19 & 170.6(2) \\ \text { Ni1-N1-C9-C4 } & 170.70(18) & \mathrm{N} 1-\mathrm{N} 2-\mathrm{C} 20-\mathrm{C} 19 & -9.7(3) \\ \mathrm{C} 1-\mathrm{N} 1-\mathrm{C} 9-\mathrm{C} 8 & 169.3(2) & \mathrm{C} 12-\mathrm{N} 2-\mathrm{C} 20-\mathrm{C} 15 & -7.3(3) \\ \text { Ni1-N1-C9-C8 } & -11.8(3) & \mathrm{N} 11-\mathrm{N} 2-\mathrm{C} 20-\mathrm{C} 15 & 172.42(18) \\ \mathrm{C} 3-\mathrm{C} 4-\mathrm{C} 9-\mathrm{N} 1 & 4.8(4) & \mathrm{C} 18-\mathrm{C} 19-\mathrm{C} 20-\mathrm{N} 2 & 178.1(2) \\ \mathrm{C} 5-\mathrm{C} 4-\mathrm{C} 9-\mathrm{N} 1 & -177.7(2) & \mathrm{P} 2-\mathrm{C} 19-\mathrm{C} 20-\mathrm{N} 2 & -8.0(3) \\ \mathrm{C} 3-\mathrm{C} 4-\mathrm{C} 9-\mathrm{C} 8 & -172.6(2) & \mathrm{C} 18-\mathrm{C} 19-\mathrm{C} 20-\mathrm{C} 15 & -4.0(4) \\ \mathrm{C} 5-\mathrm{C} 4-\mathrm{C} 9-\mathrm{C} 8 & 4.9(4) & \mathrm{P} 2-\mathrm{C} 19-\mathrm{C} 20-\mathrm{C} 15 & 169.96(18) \\ \mathrm{C} 7-\mathrm{C} 8-\mathrm{C} 9-\mathrm{N} 1 & 179.9(2) & \mathrm{C} 14-\mathrm{C} 15-\mathrm{C} 20-\mathrm{N} 2 & 5.1(4) \\ \mathrm{P} 1-\mathrm{C} 8-\mathrm{C} 9-\mathrm{N} 1 & -3.6(3) & \mathrm{C} 16-\mathrm{C} 15-\mathrm{C} 20-\mathrm{N} 2 & -176.2(2) \\ \mathrm{C} 7-\mathrm{C} 8-\mathrm{C} 9-\mathrm{C} 4 & -2.6(4) & \mathrm{C} 14-\mathrm{C} 15-\mathrm{C} 20-\mathrm{C} 19 & -172.7(2) \\ \mathrm{P} 1-\mathrm{C} 8-\mathrm{C} 9-\mathrm{C} 4 & 173.89(19) & \mathrm{C} 16-\mathrm{C} 15-\mathrm{C} 20-\mathrm{C} 19 & 6.0(4) \\ \end{array}$

(SP-4-2)-cis-Bis[8-(dimethylphosphanyl)quinoline- $\left.\kappa^{2} N, P\right]$ platinum(II) bis(tetrafluoroborate) acetonitrile monosolvate (2)

Crystal data

$\left[\mathrm{Pt}\left(\mathrm{C}_{11} \mathrm{H}_{12} \mathrm{NP}\right)_{2}\right]\left(\mathrm{BF}_{4}\right)_{2} \cdot \mathrm{C}_{2} \mathrm{H}_{3} \mathrm{~N}$

$M_{r}=788.13$

Monoclinic, $P 2{ }_{1} / n$

$a=7.9102(3) \AA$

$b=21.0833(5) \AA$

$c=16.7519$ (4) $\AA$

$\beta=95.3931(11)^{\circ}$

$V=2781.42(15) \AA^{3}$

$Z=4$

\section{Data collection}

Rigaku R-AXIS RAPID diffractometer

Radiation source: fine-focus sealed tube Detector resolution: 10.000 pixels $\mathrm{mm}^{-1}$ $\omega$ scans

Absorption correction: numerical

(NUMABS; Rigaku, 1999)

$T_{\min }=0.338, T_{\max }=0.594$

\section{Refinement}

Refinement on $F^{2}$

Least-squares matrix: full

$R\left[F^{2}>2 \sigma\left(F^{2}\right)\right]=0.033$

$w R\left(F^{2}\right)=0.084$

$S=1.07$

6350 reflections

366 parameters

0 restraints
$F(000)=1528$

$D_{\mathrm{x}}=1.882 \mathrm{Mg} \mathrm{m}^{-3}$

Mo $K \alpha$ radiation, $\lambda=0.71075 \AA$

Cell parameters from 31045 reflections

$\theta=3.1-27.5^{\circ}$

$\mu=5.23 \mathrm{~mm}^{-1}$

$T=188 \mathrm{~K}$

Platelet, colorless

$0.30 \times 0.30 \times 0.10 \mathrm{~mm}$

43967 measured reflections 6350 independent reflections

5435 reflections with $I>2 \sigma(I)$

$R_{\text {int }}=0.042$

$\theta_{\max }=27.5^{\circ}, \theta_{\min }=3.1^{\circ}$

$h=-10 \rightarrow 10$

$k=-27 \rightarrow 27$

$l=-21 \rightarrow 21$

Hydrogen site location: inferred from neighbouring sites

$\mathrm{H}$-atom parameters constrained

$w=1 /\left[\sigma^{2}\left(F_{\mathrm{o}}{ }^{2}\right)+(0.0382 P)^{2}+9.8949 P\right]$

where $P=\left(F_{\mathrm{o}}^{2}+2 F_{\mathrm{c}}^{2}\right) / 3$

$(\Delta / \sigma)_{\max }=0.001$

$\Delta \rho_{\max }=1.42 \mathrm{e} \AA^{-3}$

$\Delta \rho_{\min }=-1.44$ e $\AA^{-3}$ 


\section{Special details}

Geometry. All esds (except the esd in the dihedral angle between two 1.s. planes) are estimated using the full covariance matrix. The cell esds are taken into account individually in the estimation of esds in distances, angles and torsion angles; correlations between esds in cell parameters are only used when they are defined by crystal symmetry. An approximate (isotropic) treatment of cell esds is used for estimating esds involving l.s. planes.

Fractional atomic coordinates and isotropic or equivalent isotropic displacement parameters $\left(\AA^{2}\right)$

\begin{tabular}{|c|c|c|c|c|c|}
\hline & $x$ & $y$ & $z$ & $U_{\text {iso }} * / U_{\text {eq }}$ & Occ. $(<1)$ \\
\hline Pt1 & $0.64274(2)$ & $0.17364(2)$ & $0.20553(2)$ & $0.02183(7)$ & \\
\hline $\mathrm{P} 1$ & $0.50347(15)$ & $0.24824(5)$ & $0.26806(7)$ & $0.0237(2)$ & \\
\hline $\mathrm{P} 2$ & $0.80277(15)$ & $0.23745(6)$ & $0.13781(7)$ & $0.0247(2)$ & \\
\hline $\mathrm{F} 1$ & $1.2369(6)$ & $-0.1256(2)$ & $0.0823(5)$ & $0.136(3)$ & \\
\hline $\mathrm{F} 2 \mathrm{~A}$ & $1.5089(15)$ & $-0.1511(5)$ & $0.0808(7)$ & $0.089(2)$ & $0.573(10)$ \\
\hline F3A & $1.4191(13)$ & -0.0455 & $0.0691(7)$ & $0.089(2)$ & $0.573(10)$ \\
\hline $\mathrm{F} 4 \mathrm{~A}$ & $1.3835(12)$ & $-0.1067(4)$ & $0.1779(6)$ & $0.089(2)$ & $0.573(10)$ \\
\hline $\mathrm{F} 2 \mathrm{~B}$ & $1.4392(16)$ & $-0.1049(8)$ & $0.0213(10)$ & $0.121(4)$ & $0.427(10)$ \\
\hline F3B & $1.4100(18)$ & $-0.0423(9)$ & $0.1216(12)$ & $0.121(4)$ & $0.427(10)$ \\
\hline F4B & $1.520(2)$ & $-0.1430(9)$ & $0.1262(12)$ & $0.121(4)$ & $0.427(10)$ \\
\hline F5 & $0.0397(6)$ & $0.3460(3)$ & $-0.0167(5)$ & $0.115(2)$ & \\
\hline F6 & $0.2266(7)$ & $0.4071(2)$ & $-0.0644(3)$ & $0.0883(15)$ & \\
\hline F7 & $0.1968(10)$ & $0.4023(3)$ & $0.0675(3)$ & $0.126(3)$ & \\
\hline F8 & $0.3139(5)$ & $0.31996(17)$ & $0.0067(2)$ & $0.0566(10)$ & \\
\hline N1 & $0.4883(5)$ & $0.11161(18)$ & $0.2672(2)$ & $0.0256(8)$ & \\
\hline $\mathrm{N} 2$ & $0.7738(5)$ & $0.10240(18)$ & $0.1453(2)$ & $0.0270(8)$ & \\
\hline N3 & $0.8437(7)$ & $-0.0443(3)$ & $0.3316(4)$ & $0.0521(13)$ & \\
\hline $\mathrm{C} 1$ & $0.5191(7)$ & $0.0503(2)$ & $0.2795(3)$ & $0.0342(11)$ & \\
\hline $\mathrm{H} 1$ & 0.6285 & 0.0343 & 0.2714 & $0.041^{*}$ & \\
\hline $\mathrm{C} 2$ & $0.3965(8)$ & $0.0078(3)$ & $0.3041(3)$ & $0.0411(13)$ & \\
\hline $\mathrm{H} 2$ & 0.4219 & -0.0361 & 0.3103 & $0.049^{*}$ & \\
\hline $\mathrm{C} 3$ & $0.2415(7)$ & $0.0304(3)$ & $0.3190(3)$ & $0.0395(12)$ & \\
\hline H3 & 0.1547 & 0.0020 & 0.3317 & $0.047^{*}$ & \\
\hline $\mathrm{C} 4$ & $0.2103(6)$ & $0.0963(2)$ & $0.3155(3)$ & $0.0310(10)$ & \\
\hline $\mathrm{C} 5$ & $0.0599(6)$ & $0.1251(3)$ & $0.3391(3)$ & $0.0350(11)$ & \\
\hline H5 & -0.0327 & 0.0992 & 0.3512 & $0.042 *$ & \\
\hline C6 & $0.0477(7)$ & $0.1893(3)$ & $0.3444(3)$ & $0.0346(11)$ & \\
\hline H6 & -0.0519 & 0.2077 & 0.3620 & $0.042 *$ & \\
\hline $\mathrm{C} 7$ & $0.1812(6)$ & 0.2289 (2) & 0.3239 (3) & $0.0307(10)$ & \\
\hline $\mathrm{H} 7$ & 0.1727 & 0.2736 & 0.3298 & $0.037^{*}$ & \\
\hline $\mathrm{C} 8$ & $0.3228(6)$ & $0.2031(2)$ & $0.2955(3)$ & $0.0253(9)$ & \\
\hline C9 & $0.3391(6)$ & $0.1368(2)$ & $0.2909(3)$ & $0.0254(9)$ & \\
\hline $\mathrm{C} 10$ & $0.6062(6)$ & 0.2807 (2) & 0.3599 (3) & $0.0328(11)$ & \\
\hline H10A & 0.6548 & 0.2461 & 0.3938 & $0.039^{*}$ & \\
\hline H10B & 0.6968 & 0.3097 & 0.3474 & $0.039^{*}$ & \\
\hline $\mathrm{H} 10 \mathrm{C}$ & 0.5227 & 0.3038 & 0.3884 & $0.039^{*}$ & \\
\hline $\mathrm{C} 11$ & $0.4207(7)$ & $0.3163(2)$ & $0.2120(3)$ & $0.0353(11)$ & \\
\hline H11A & 0.3625 & 0.3023 & 0.1609 & $0.042^{*}$ & \\
\hline H11B & 0.3403 & 0.3389 & 0.2429 & $0.042 *$ & \\
\hline
\end{tabular}




$\begin{array}{lllll}\text { H11C } & 0.5144 & 0.3447 & 0.2019 & 0.042^{*} \\ \text { C12 } & 0.7232(7) & 0.0429(2) & 0.1333(3) & 0.0333(11) \\ \text { H12 } & 0.6088 & 0.0325 & 0.1405 & 0.040^{*} \\ \text { C13 } & 0.8316(7) & -0.0059(2) & 0.1104(3) & 0.0380(12) \\ \text { H13 } & 0.7911 & -0.0482 & 0.1044 & 0.046^{*} \\ \text { C14 } & 0.9946(7) & 0.0083(3) & 0.0970(3) & 0.0392(12) \\ \text { H14 } & 1.0717 & -0.0244 & 0.0861 & 0.047^{*} \\ \text { C15 } & 1.0478(6) & 0.0725(2) & 0.0996(3) & 0.0311(10) \\ \text { C16 } & 1.2074(6) & 0.0932(3) & 0.0757(3) & 0.0392(12) \\ \text { H16 } & 1.2897 & 0.0628 & 0.0632 & 0.047^{*} \\ \text { C17 } & 1.2421(7) & 0.1564(3) & 0.0707(3) & 0.0409(13) \\ \text { H17 } & 1.3472 & 0.1696 & 0.0530 & 0.049^{*} \\ \text { C18 } & 1.1258(6) & 0.2021(3) & 0.0913(3) & 0.0350(11) \\ \text { H18 } & 1.1518 & 0.2459 & 0.0865 & 0.042^{*} \\ \text { C19 } & 0.9729(6) & 0.1843(2) & 0.1186(3) & 0.0268(10) \\ \text { C20 } & 0.9326(6) & 0.1191(2) & 0.1230(3) & 0.0256(9) \\ \text { C21 } & 0.8949(7) & 0.3084(2) & 0.1834(3) & 0.0349(11) \\ \text { H21A } & 0.8043 & 0.3379 & 0.1944 & 0.042^{*} \\ \text { H21B } & 0.9617 & 0.2974 & 0.2338 & 0.042^{*} \\ \text { H21C } & 0.9688 & 0.3284 & 0.1469 & 0.042^{*} \\ \text { C22 } & 0.7056(7) & 0.2620(3) & 0.0406(3) & 0.0354(11) \\ \text { H22A } & 0.6527 & 0.2252 & 0.0124 & 0.042^{*} \\ \text { H22B } & 0.6189 & 0.2942 & 0.0476 & 0.042^{*} \\ \text { H22C } & 0.7926 & 0.2798 & 0.0091 & 0.042^{*} \\ \text { C23 } & 0.8162(8) & -0.0480(3) & 0.3952(4) & 0.0437(13) \\ \text { C24 } & 0.7834(13) & -0.0553(4) & 0.4779(4) & 0.078(2) \\ \text { H24A } & 0.7717 & -0.0134 & 0.5021 & 0.094^{*} \\ \text { H24B } & 0.8781 & -0.0780 & 0.5071 & 0.094^{*} \\ \text { H24C } & 0.6783 & -0.0794 & 0.4808 & 0.094^{*} \\ \text { B1 } & 1.3953(8) & -0.1035(3) & 0.0979(4) & 0.0361(13) \\ \text { B2 } & 0.2039(8) & 0.3716(3) & 0.0001(4) & 0.0370(13) \\ & & & & \end{array}$

Atomic displacement parameters $\left(\AA^{2}\right)$

\begin{tabular}{lllllll}
\hline & $U^{11}$ & $U^{22}$ & $U^{33}$ & $U^{12}$ & $U^{13}$ & $U^{23}$ \\
\hline Pt1 & $0.02079(9)$ & $0.02222(10)$ & $0.02284(10)$ & $0.00020(6)$ & $0.00396(6)$ & $0.00096(7)$ \\
P1 & $0.0234(6)$ & $0.0233(5)$ & $0.0243(6)$ & $0.0018(4)$ & $0.0025(4)$ & $0.0014(5)$ \\
P2 & $0.0221(6)$ & $0.0261(6)$ & $0.0258(6)$ & $-0.0021(4)$ & $0.0023(4)$ & $0.0032(5)$ \\
F1 & $0.040(2)$ & $0.051(3)$ & $0.317(10)$ & $-0.003(2)$ & $0.017(4)$ & $0.009(4)$ \\
F2A & $0.104(4)$ & $0.057(3)$ & $0.106(5)$ & $0.009(3)$ & $0.014(4)$ & $0.015(3)$ \\
F3A & $0.104(4)$ & $0.057(3)$ & $0.106(5)$ & $0.009(3)$ & $0.014(4)$ & $0.015(3)$ \\
F4A & $0.104(4)$ & $0.057(3)$ & $0.106(5)$ & $0.009(3)$ & $0.014(4)$ & $0.015(3)$ \\
F2B & $0.071(5)$ & $0.136(8)$ & $0.158(10)$ & $-0.024(5)$ & $0.031(6)$ & $-0.015(7)$ \\
F3B & $0.071(5)$ & $0.136(8)$ & $0.158(10)$ & $-0.024(5)$ & $0.031(6)$ & $-0.015(7)$ \\
F4B & $0.071(5)$ & $0.136(8)$ & $0.158(10)$ & $-0.024(5)$ & $0.031(6)$ & $-0.015(7)$ \\
F5 & $0.052(3)$ & $0.081(3)$ & $0.215(7)$ & $0.001(2)$ & $0.032(4)$ & $0.012(4)$ \\
F6 & $0.108(4)$ & $0.087(3)$ & $0.075(3)$ & $0.032(3)$ & $0.037(3)$ & $0.040(3)$ \\
F7 & $0.207(7)$ & $0.110(4)$ & $0.057(3)$ & $0.077(5)$ & $-0.008(4)$ & $-0.027(3)$
\end{tabular}




$\begin{array}{lllllll}\text { F8 } & 0.053(2) & 0.054(2) & 0.062(2) & 0.0172(17) & 0.0060(18) & 0.0040(18) \\ \text { N1 } & 0.0241(19) & 0.0264(19) & 0.0269(19) & 0.0009(15) & 0.0066(15) & 0.0044(16) \\ \text { N2 } & 0.028(2) & 0.027(2) & 0.0252(19) & -0.0004(16) & 0.0028(16) & -0.0020(16) \\ \text { N3 } & 0.052(3) & 0.049(3) & 0.057(3) & 0.003(2) & 0.013(3) & 0.004(3) \\ \text { C1 } & 0.040(3) & 0.028(2) & 0.037(3) & 0.004(2) & 0.012(2) & 0.004(2) \\ \text { C2 } & 0.058(4) & 0.026(3) & 0.042(3) & 0.000(2) & 0.018(3) & 0.006(2) \\ \text { C3 } & 0.045(3) & 0.034(3) & 0.042(3) & -0.010(2) & 0.014(2) & 0.003(2) \\ \text { C4 } & 0.030(2) & 0.037(3) & 0.026(2) & -0.005(2) & 0.0045(19) & 0.000(2) \\ \text { C5 } & 0.025(2) & 0.049(3) & 0.032(3) & -0.003(2) & 0.007(2) & 0.004(2) \\ \text { C6 } & 0.030(3) & 0.047(3) & 0.028(2) & 0.009(2) & 0.006(2) & 0.005(2) \\ \text { C7 } & 0.030(2) & 0.038(3) & 0.025(2) & 0.006(2) & 0.0046(19) & 0.001(2) \\ \text { C8 } & 0.026(2) & 0.031(2) & 0.019(2) & 0.0008(18) & 0.0018(17) & 0.0029(18) \\ \text { C9 } & 0.026(2) & 0.029(2) & 0.022(2) & 0.0007(18) & 0.0033(18) & 0.0014(18) \\ \text { C10 } & 0.032(3) & 0.036(3) & 0.030(2) & 0.000(2) & 0.001(2) & -0.007(2) \\ \text { C11 } & 0.033(3) & 0.031(3) & 0.043(3) & 0.004(2) & 0.006(2) & 0.009(2) \\ \text { C12 } & 0.035(3) & 0.030(2) & 0.036(3) & -0.004(2) & 0.010(2) & -0.004(2) \\ \text { C13 } & 0.052(3) & 0.025(2) & 0.038(3) & 0.000(2) & 0.013(2) & -0.004(2) \\ \text { C14 } & 0.047(3) & 0.038(3) & 0.033(3) & 0.013(2) & 0.007(2) & -0.002(2) \\ \text { C15 } & 0.029(2) & 0.039(3) & 0.025(2) & 0.006(2) & 0.0046(19) & -0.003(2) \\ \text { C16 } & 0.026(3) & 0.057(3) & 0.034(3) & 0.009(2) & 0.004(2) & -0.004(3) \\ \text { C17 } & 0.026(3) & 0.062(4) & 0.035(3) & -0.007(2) & 0.007(2) & -0.010(3) \\ \text { C18 } & 0.028(2) & 0.045(3) & 0.033(3) & -0.007(2) & 0.008(2) & -0.003(2) \\ \text { C19 } & 0.024(2) & 0.033(2) & 0.024(2) & -0.0016(18) & 0.0028(18) & -0.0035(19) \\ \text { C20 } & 0.022(2) & 0.031(2) & 0.023(2) & 0.0034(18) & 0.0017(17) & 0.0004(19) \\ \text { C21 } & 0.030(3) & 0.029(2) & 0.046(3) & -0.004(2) & 0.001(2) & -0.002(2) \\ \text { C22 } & 0.034(3) & 0.043(3) & 0.029(2) & -0.001(2) & 0.000(2) & 0.007(2) \\ \text { C23 } & 0.044(3) & 0.030(3) & 0.057(4) & -0.006(2) & 0.005(3) & -0.001(3) \\ \text { C24 } & 0.125(7) & 0.057(4) & 0.054(4) & -0.025(5) & 0.020(5) & 0.005(4) \\ \text { B1 } & 0.038(3) & 0.028(3) & 0.044(4) & 0.003(2) & 0.010(3) & -0.002(3) \\ \text { B2 } & 0.043(3) & 0.034(3) & 0.036(3) & 0.005(3) & 0.014(3) & 0.001(3)\end{array}$

Geometric parameters $\left(A,{ }^{\circ}\right)$

\begin{tabular}{llll}
\hline Pt1-N1 & $2.123(4)$ & $\mathrm{C} 6-\mathrm{C} 7$ & $1.414(7)$ \\
Pt1-N2 & $2.132(4)$ & $\mathrm{C} 6-\mathrm{H} 6$ & 0.9500 \\
Pt1-P2 & $2.2293(12)$ & $\mathrm{C} 7-\mathrm{C} 8$ & $1.371(6)$ \\
Pt1-P1 & $2.2365(12)$ & $\mathrm{C} 7-\mathrm{H} 7$ & 0.9500 \\
P1-C11 & $1.804(5)$ & $\mathrm{C} 8-\mathrm{C} 9$ & $1.405(7)$ \\
P1-C10 & $1.805(5)$ & $\mathrm{C} 10-\mathrm{H} 10 \mathrm{~A}$ & 0.9800 \\
P1-C8 & $1.812(5)$ & $\mathrm{C} 10-\mathrm{H} 10 \mathrm{~B}$ & 0.9800 \\
P2-C21 & $1.802(5)$ & $\mathrm{C} 10-\mathrm{H} 10 \mathrm{C}$ & 0.9800 \\
P2-C19 & $1.804(5)$ & $\mathrm{C} 11-\mathrm{H} 11 \mathrm{~A}$ & 0.9800 \\
P2-C22 & $1.810(5)$ & $\mathrm{C} 11-\mathrm{H} 11 \mathrm{~B}$ & 0.9800 \\
F1-B1 & $1.340(8)$ & $\mathrm{C} 11-\mathrm{H} 11 \mathrm{C}$ & 0.9800 \\
F2A-B1 & $1.395(13)$ & $\mathrm{C} 12-\mathrm{C} 13$ & $1.416(7)$ \\
F3A-B1 & $1.334(11)$ & $\mathrm{C} 12-\mathrm{H} 12$ & 0.9500 \\
F4A-B1 & $1.353(11)$ & $\mathrm{C} 13-\mathrm{C} 14$ & $1.363(8)$ \\
F2B-B1 & $1.361(17)$ & $\mathrm{C} 13-\mathrm{H} 13$ & 0.9500
\end{tabular}




\begin{tabular}{|c|c|c|c|}
\hline $\mathrm{F} 3 \mathrm{~B}-\mathrm{B} 1$ & $1.351(18)$ & $\mathrm{C} 14-\mathrm{C} 15$ & $1.416(8)$ \\
\hline $\mathrm{F} 4 \mathrm{~B}-\mathrm{B} 1$ & $1.35(2)$ & $\mathrm{C} 14-\mathrm{H} 14$ & 0.9500 \\
\hline $\mathrm{F} 5-\mathrm{B} 2$ & $1.410(8)$ & $\mathrm{C} 15-\mathrm{C} 20$ & $1.421(6)$ \\
\hline $\mathrm{F} 6-\mathrm{B} 2$ & $1.341(7)$ & $\mathrm{C} 15-\mathrm{C} 16$ & $1.428(7)$ \\
\hline F7-B2 & $1.309(8)$ & $\mathrm{C} 16-\mathrm{C} 17$ & $1.366(8)$ \\
\hline $\mathrm{F} 8-\mathrm{B} 2$ & $1.391(7)$ & $\mathrm{C} 16-\mathrm{H} 16$ & 0.9500 \\
\hline $\mathrm{N} 1-\mathrm{C} 1$ & $1.329(6)$ & $\mathrm{C} 17-\mathrm{C} 18$ & $1.398(8)$ \\
\hline $\mathrm{N} 1-\mathrm{C} 9$ & $1.386(6)$ & $\mathrm{C} 17-\mathrm{H} 17$ & 0.9500 \\
\hline $\mathrm{N} 2-\mathrm{C} 12$ & $1.326(6)$ & $\mathrm{C} 18-\mathrm{C} 19$ & $1.384(7)$ \\
\hline $\mathrm{N} 2-\mathrm{C} 20$ & $1.389(6)$ & $\mathrm{C} 18-\mathrm{H} 18$ & 0.9500 \\
\hline $\mathrm{N} 3-\mathrm{C} 23$ & $1.110(8)$ & $\mathrm{C} 19-\mathrm{C} 20$ & $1.414(6)$ \\
\hline $\mathrm{C} 1-\mathrm{C} 2$ & $1.409(7)$ & $\mathrm{C} 21-\mathrm{H} 21 \mathrm{~A}$ & 0.9800 \\
\hline $\mathrm{C} 1-\mathrm{H} 1$ & 0.9500 & $\mathrm{C} 21-\mathrm{H} 21 \mathrm{~B}$ & 0.9800 \\
\hline $\mathrm{C} 2-\mathrm{C} 3$ & $1.360(8)$ & $\mathrm{C} 21-\mathrm{H} 21 \mathrm{C}$ & 0.9800 \\
\hline $\mathrm{C} 2-\mathrm{H} 2$ & 0.9500 & $\mathrm{C} 22-\mathrm{H} 22 \mathrm{~A}$ & 0.9800 \\
\hline $\mathrm{C} 3-\mathrm{C} 4$ & $1.410(7)$ & $\mathrm{C} 22-\mathrm{H} 22 \mathrm{~B}$ & 0.9800 \\
\hline $\mathrm{C} 3-\mathrm{H} 3$ & 0.9500 & $\mathrm{C} 22-\mathrm{H} 22 \mathrm{C}$ & 0.9800 \\
\hline $\mathrm{C} 4-\mathrm{C} 9$ & $1.421(7)$ & $\mathrm{C} 23-\mathrm{C} 24$ & $1.441(9)$ \\
\hline $\mathrm{C} 4-\mathrm{C} 5$ & $1.424(7)$ & $\mathrm{C} 24-\mathrm{H} 24 \mathrm{~A}$ & 0.9800 \\
\hline $\mathrm{C} 5-\mathrm{C} 6$ & $1.361(8)$ & $\mathrm{C} 24-\mathrm{H} 24 \mathrm{~B}$ & 0.9800 \\
\hline $\mathrm{C} 5-\mathrm{H} 5$ & 0.9500 & $\mathrm{C} 24-\mathrm{H} 24 \mathrm{C}$ & 0.9800 \\
\hline $\mathrm{N} 1-\mathrm{Pt} 1-\mathrm{N} 2$ & $97.13(15)$ & $\mathrm{N} 2-\mathrm{C} 12-\mathrm{H} 12$ & 118.4 \\
\hline $\mathrm{N} 1-\mathrm{Pt} 1-\mathrm{P} 2$ & $178.51(11)$ & $\mathrm{C} 13-\mathrm{C} 12-\mathrm{H} 12$ & 118.4 \\
\hline $\mathrm{N} 2-\mathrm{Pt} 1-\mathrm{P} 2$ & $81.93(11)$ & $\mathrm{C} 14-\mathrm{C} 13-\mathrm{C} 12$ & $119.4(5)$ \\
\hline $\mathrm{N} 1-\mathrm{Pt} 1-\mathrm{P} 1$ & $82.76(11)$ & $\mathrm{C} 14-\mathrm{C} 13-\mathrm{H} 13$ & 120.3 \\
\hline $\mathrm{N} 2-\mathrm{Pt} 1-\mathrm{P} 1$ & $179.55(11)$ & $\mathrm{C} 12-\mathrm{C} 13-\mathrm{H} 13$ & 120.3 \\
\hline $\mathrm{P} 2-\mathrm{Pt} 1-\mathrm{P} 1$ & $98.17(4)$ & $\mathrm{C} 13-\mathrm{C} 14-\mathrm{C} 15$ & $119.2(5)$ \\
\hline $\mathrm{C} 11-\mathrm{P} 1-\mathrm{C} 10$ & $104.7(3)$ & $\mathrm{C} 13-\mathrm{C} 14-\mathrm{H} 14$ & 120.4 \\
\hline $\mathrm{C} 11-\mathrm{P} 1-\mathrm{C} 8$ & $107.1(2)$ & $\mathrm{C} 15-\mathrm{C} 14-\mathrm{H} 14$ & 120.4 \\
\hline $\mathrm{C} 10-\mathrm{P} 1-\mathrm{C} 8$ & $106.6(2)$ & $\mathrm{C} 14-\mathrm{C} 15-\mathrm{C} 20$ & $118.3(5)$ \\
\hline $\mathrm{C} 11-\mathrm{P} 1-\mathrm{Pt} 1$ & $119.12(19)$ & $\mathrm{C} 14-\mathrm{C} 15-\mathrm{C} 16$ & $123.4(5)$ \\
\hline $\mathrm{C} 10-\mathrm{P} 1-\mathrm{Pt} 1$ & $117.60(17)$ & $\mathrm{C} 20-\mathrm{C} 15-\mathrm{C} 16$ & $118.2(5)$ \\
\hline $\mathrm{C} 8-\mathrm{P} 1-\mathrm{Pt} 1$ & $100.60(16)$ & $\mathrm{C} 17-\mathrm{C} 16-\mathrm{C} 15$ & $120.2(5)$ \\
\hline $\mathrm{C} 21-\mathrm{P} 2-\mathrm{C} 19$ & $108.2(2)$ & $\mathrm{C} 17-\mathrm{C} 16-\mathrm{H} 16$ & 119.9 \\
\hline $\mathrm{C} 21-\mathrm{P} 2-\mathrm{C} 22$ & $105.4(3)$ & $\mathrm{C} 15-\mathrm{C} 16-\mathrm{H} 16$ & 119.9 \\
\hline $\mathrm{C} 19-\mathrm{P} 2-\mathrm{C} 22$ & $106.1(2)$ & $\mathrm{C} 16-\mathrm{C} 17-\mathrm{C} 18$ & $121.2(5)$ \\
\hline $\mathrm{C} 21-\mathrm{P} 2-\mathrm{Pt} 1$ & $120.76(19)$ & $\mathrm{C} 16-\mathrm{C} 17-\mathrm{H} 17$ & 119.4 \\
\hline $\mathrm{C} 19-\mathrm{P} 2-\mathrm{Pt} 1$ & $100.65(16)$ & $\mathrm{C} 18-\mathrm{C} 17-\mathrm{H} 17$ & 119.4 \\
\hline $\mathrm{C} 22-\mathrm{P} 2-\mathrm{Pt} 1$ & $114.64(18)$ & $\mathrm{C} 19-\mathrm{C} 18-\mathrm{C} 17$ & $120.6(5)$ \\
\hline $\mathrm{C} 1-\mathrm{N} 1-\mathrm{C} 9$ & 118.5 & $\mathrm{C} 19-\mathrm{C} 18-\mathrm{H} 18$ & 119.7 \\
\hline $\mathrm{C} 1-\mathrm{N} 1-\mathrm{Pt} 1$ & $124.7(3)$ & $\mathrm{C} 17-\mathrm{C} 18-\mathrm{H} 18$ & 119.7 \\
\hline $\mathrm{C} 9-\mathrm{N} 1-\mathrm{Pt} 1$ & $116.6(3)$ & $\mathrm{C} 18-\mathrm{C} 19-\mathrm{C} 20$ & $119.3(4)$ \\
\hline $\mathrm{C} 12-\mathrm{N} 2-\mathrm{C} 20$ & $117.8(4)$ & $\mathrm{C} 18-\mathrm{C} 19-\mathrm{P} 2$ & $125.4(4)$ \\
\hline $\mathrm{C} 12-\mathrm{N} 2-\mathrm{Pt} 1$ & 125.8 & $\mathrm{C} 20-\mathrm{C} 19-\mathrm{P} 2$ & $114.7(3)$ \\
\hline $\mathrm{C} 20-\mathrm{N} 2-\mathrm{Pt} 1$ & $116.2(3)$ & $\mathrm{N} 2-\mathrm{C} 20-\mathrm{C} 19$ & $118.3(4)$ \\
\hline $\mathrm{N} 1-\mathrm{C} 1-\mathrm{C} 2$ & $122.9(5)$ & $\mathrm{N} 2-\mathrm{C} 20-\mathrm{C} 15$ & $121.2(4)$ \\
\hline $\mathrm{N} 1-\mathrm{C} 1-\mathrm{H} 1$ & 118.6 & $\mathrm{C} 19-\mathrm{C} 20-\mathrm{C} 15$ & $120.3(4)$ \\
\hline
\end{tabular}




\begin{tabular}{|c|c|c|c|}
\hline $\mathrm{C} 2-\mathrm{C} 1-\mathrm{H} 1$ & 118.6 & $\mathrm{P} 2-\mathrm{C} 21-\mathrm{H} 21 \mathrm{~A}$ & 109.5 \\
\hline $\mathrm{C} 3-\mathrm{C} 2-\mathrm{C} 1$ & $119.2(5)$ & $\mathrm{P} 2-\mathrm{C} 21-\mathrm{H} 21 \mathrm{~B}$ & 109.5 \\
\hline $\mathrm{C} 3-\mathrm{C} 2-\mathrm{H} 2$ & 120.4 & $\mathrm{H} 21 \mathrm{~A}-\mathrm{C} 21-\mathrm{H} 21 \mathrm{~B}$ & 109.5 \\
\hline $\mathrm{C} 1-\mathrm{C} 2-\mathrm{H} 2$ & 120.4 & $\mathrm{P} 2-\mathrm{C} 21-\mathrm{H} 21 \mathrm{C}$ & 109.5 \\
\hline $\mathrm{C} 2-\mathrm{C} 3-\mathrm{C} 4$ & $119.7(5)$ & $\mathrm{H} 21 \mathrm{~A}-\mathrm{C} 21-\mathrm{H} 21 \mathrm{C}$ & 109.5 \\
\hline $\mathrm{C} 2-\mathrm{C} 3-\mathrm{H} 3$ & 120.2 & $\mathrm{H} 21 \mathrm{~B}-\mathrm{C} 21-\mathrm{H} 21 \mathrm{C}$ & 109.5 \\
\hline $\mathrm{C} 4-\mathrm{C} 3-\mathrm{H} 3$ & 120.2 & $\mathrm{P} 2-\mathrm{C} 22-\mathrm{H} 22 \mathrm{~A}$ & 109.5 \\
\hline $\mathrm{C} 3-\mathrm{C} 4-\mathrm{C} 9$ & $118.5(5)$ & $\mathrm{P} 2-\mathrm{C} 22-\mathrm{H} 22 \mathrm{~B}$ & 109.5 \\
\hline $\mathrm{C} 3-\mathrm{C} 4-\mathrm{C} 5$ & $123.8(5)$ & $\mathrm{H} 22 \mathrm{~A}-\mathrm{C} 22-\mathrm{H} 22 \mathrm{~B}$ & 109.5 \\
\hline $\mathrm{C} 9-\mathrm{C} 4-\mathrm{C} 5$ & $117.6(5)$ & $\mathrm{P} 2-\mathrm{C} 22-\mathrm{H} 22 \mathrm{C}$ & 109.5 \\
\hline $\mathrm{C} 6-\mathrm{C} 5-\mathrm{C} 4$ & $120.6(5)$ & $\mathrm{H} 22 \mathrm{~A}-\mathrm{C} 22-\mathrm{H} 22 \mathrm{C}$ & 109.5 \\
\hline $\mathrm{C} 6-\mathrm{C} 5-\mathrm{H} 5$ & 119.7 & $\mathrm{H} 22 \mathrm{~B}-\mathrm{C} 22-\mathrm{H} 22 \mathrm{C}$ & 109.5 \\
\hline $\mathrm{C} 4-\mathrm{C} 5-\mathrm{H} 5$ & 119.7 & $\mathrm{~N} 3-\mathrm{C} 23-\mathrm{C} 24$ & $177.7(7)$ \\
\hline $\mathrm{C} 5-\mathrm{C} 6-\mathrm{C} 7$ & $120.8(5)$ & $\mathrm{C} 23-\mathrm{C} 24-\mathrm{H} 24 \mathrm{~A}$ & 109.5 \\
\hline $\mathrm{C} 5-\mathrm{C} 6-\mathrm{H} 6$ & 119.6 & $\mathrm{C} 23-\mathrm{C} 24-\mathrm{H} 24 \mathrm{~B}$ & 109.5 \\
\hline $\mathrm{C} 7-\mathrm{C} 6-\mathrm{H} 6$ & 119.6 & $\mathrm{H} 24 \mathrm{~A}-\mathrm{C} 24-\mathrm{H} 24 \mathrm{~B}$ & 109.5 \\
\hline $\mathrm{C} 8-\mathrm{C} 7-\mathrm{C} 6$ & $120.2(5)$ & $\mathrm{C} 23-\mathrm{C} 24-\mathrm{H} 24 \mathrm{C}$ & 109.5 \\
\hline $\mathrm{C} 8-\mathrm{C} 7-\mathrm{H} 7$ & 119.9 & $\mathrm{H} 24 \mathrm{~A}-\mathrm{C} 24-\mathrm{H} 24 \mathrm{C}$ & 109.5 \\
\hline $\mathrm{C} 6-\mathrm{C} 7-\mathrm{H} 7$ & 119.9 & $\mathrm{H} 24 \mathrm{~B}-\mathrm{C} 24-\mathrm{H} 24 \mathrm{C}$ & 109.5 \\
\hline $\mathrm{C} 7-\mathrm{C} 8-\mathrm{C} 9$ & $119.8(4)$ & $\mathrm{F} 3 \mathrm{~A}-\mathrm{B} 1-\mathrm{F} 1$ & $114.2(7)$ \\
\hline $\mathrm{C} 7-\mathrm{C} 8-\mathrm{P} 1$ & $124.7(4)$ & $\mathrm{F} 1-\mathrm{B} 1-\mathrm{F} 4 \mathrm{~B}$ & $119.8(9)$ \\
\hline $\mathrm{C} 9-\mathrm{C} 8-\mathrm{P} 1$ & $115.5(3)$ & $\mathrm{F} 1-\mathrm{B} 1-\mathrm{F} 3 \mathrm{~B}$ & $116.3(8)$ \\
\hline $\mathrm{N} 1-\mathrm{C} 9-\mathrm{C} 8$ & $118.8(4)$ & $\mathrm{F} 4 \mathrm{~B}-\mathrm{B} 1-\mathrm{F} 3 \mathrm{~B}$ & $116.6(12)$ \\
\hline $\mathrm{N} 1-\mathrm{C} 9-\mathrm{C} 4$ & $120.3(4)$ & $\mathrm{F} 3 \mathrm{~A}-\mathrm{B} 1-\mathrm{F} 4 \mathrm{~A}$ & $115.4(8)$ \\
\hline $\mathrm{C} 8-\mathrm{C} 9-\mathrm{C} 4$ & $120.7(4)$ & $\mathrm{F} 1-\mathrm{B} 1-\mathrm{F} 4 \mathrm{~A}$ & $91.4(7)$ \\
\hline $\mathrm{P} 1-\mathrm{C} 10-\mathrm{H} 10 \mathrm{~A}$ & 109.5 & $\mathrm{~F} 1-\mathrm{B} 1-\mathrm{F} 2 \mathrm{~B}$ & $97.2(8)$ \\
\hline $\mathrm{P} 1-\mathrm{C} 10-\mathrm{H} 10 \mathrm{~B}$ & 109.5 & $\mathrm{~F} 4 \mathrm{~B}-\mathrm{B} 1-\mathrm{F} 2 \mathrm{~B}$ & $94.3(11)$ \\
\hline $\mathrm{H} 10 \mathrm{~A}-\mathrm{C} 10-\mathrm{H} 10 \mathrm{~B}$ & 109.5 & $\mathrm{~F} 3 \mathrm{~B}-\mathrm{B} 1-\mathrm{F} 2 \mathrm{~B}$ & $106.0(11)$ \\
\hline $\mathrm{P} 1-\mathrm{C} 10-\mathrm{H} 10 \mathrm{C}$ & 109.5 & $\mathrm{~F} 3 \mathrm{~A}-\mathrm{B} 1-\mathrm{F} 2 \mathrm{~A}$ & $118.0(8)$ \\
\hline $\mathrm{H} 10 \mathrm{~A}-\mathrm{C} 10-\mathrm{H} 10 \mathrm{C}$ & 109.5 & $\mathrm{~F} 1-\mathrm{B} 1-\mathrm{F} 2 \mathrm{~A}$ & $108.5(7)$ \\
\hline $\mathrm{H} 10 \mathrm{~B}-\mathrm{C} 10-\mathrm{H} 10 \mathrm{C}$ & 109.5 & $\mathrm{~F} 4 \mathrm{~A}-\mathrm{B} 1-\mathrm{F} 2 \mathrm{~A}$ & $105.8(7)$ \\
\hline $\mathrm{P} 1-\mathrm{C} 11-\mathrm{H} 11 \mathrm{~A}$ & 109.5 & $\mathrm{~F} 7-\mathrm{B} 2-\mathrm{F} 6$ & $116.0(6)$ \\
\hline $\mathrm{P} 1-\mathrm{C} 11-\mathrm{H} 11 \mathrm{~B}$ & 109.5 & $\mathrm{~F} 7-\mathrm{B} 2-\mathrm{F} 8$ & $113.3(6)$ \\
\hline $\mathrm{H} 11 \mathrm{~A}-\mathrm{C} 11-\mathrm{H} 11 \mathrm{~B}$ & 109.5 & $\mathrm{~F} 6-\mathrm{B} 2-\mathrm{F} 8$ & $111.8(5)$ \\
\hline $\mathrm{P} 1-\mathrm{C} 11-\mathrm{H} 11 \mathrm{C}$ & 109.5 & $\mathrm{~F} 7-\mathrm{B} 2-\mathrm{F} 5$ & $104.4(6)$ \\
\hline $\mathrm{H} 11 \mathrm{~A}-\mathrm{C} 11-\mathrm{H} 11 \mathrm{C}$ & 109.5 & $\mathrm{~F} 6-\mathrm{B} 2-\mathrm{F} 5$ & $104.1(6)$ \\
\hline $\mathrm{H} 11 \mathrm{~B}-\mathrm{C} 11-\mathrm{H} 11 \mathrm{C}$ & 109.5 & $\mathrm{~F} 8-\mathrm{B} 2-\mathrm{F} 5$ & $105.9(5)$ \\
\hline $\mathrm{N} 2-\mathrm{C} 12-\mathrm{C} 13$ & $123.2(5)$ & & \\
\hline $\mathrm{C} 9-\mathrm{N} 1-\mathrm{C} 1-\mathrm{C} 2$ & $10.0(7)$ & $\mathrm{C} 20-\mathrm{N} 2-\mathrm{C} 12-\mathrm{C} 13$ & $9.6(7)$ \\
\hline $\mathrm{Pt} 1-\mathrm{N} 1-\mathrm{C} 1-\mathrm{C} 2$ & $-165.3(4)$ & $\mathrm{Pt} 1-\mathrm{N} 2-\mathrm{C} 12-\mathrm{C} 13$ & $-164.6(4)$ \\
\hline $\mathrm{N} 1-\mathrm{C} 1-\mathrm{C} 2-\mathrm{C} 3$ & $-2.5(8)$ & $\mathrm{N} 2-\mathrm{C} 12-\mathrm{C} 13-\mathrm{C} 14$ & $-2.3(8)$ \\
\hline $\mathrm{C} 1-\mathrm{C} 2-\mathrm{C} 3-\mathrm{C} 4$ & $-4.8(8)$ & $\mathrm{C} 12-\mathrm{C} 13-\mathrm{C} 14-\mathrm{C} 15$ & $-5.4(8)$ \\
\hline $\mathrm{C} 2-\mathrm{C} 3-\mathrm{C} 4-\mathrm{C} 9$ & $4.3(8)$ & $\mathrm{C} 13-\mathrm{C} 14-\mathrm{C} 15-\mathrm{C} 20$ & $5.4(7)$ \\
\hline $\mathrm{C} 2-\mathrm{C} 3-\mathrm{C} 4-\mathrm{C} 5$ & $-172.4(5)$ & $\mathrm{C} 13-\mathrm{C} 14-\mathrm{C} 15-\mathrm{C} 16$ & $-171.1(5)$ \\
\hline $\mathrm{C} 3-\mathrm{C} 4-\mathrm{C} 5-\mathrm{C} 6$ & $171.1(5)$ & $\mathrm{C} 14-\mathrm{C} 15-\mathrm{C} 16-\mathrm{C} 17$ & $172.3(5)$ \\
\hline $\mathrm{C} 9-\mathrm{C} 4-\mathrm{C} 5-\mathrm{C} 6$ & $-5.6(7)$ & $\mathrm{C} 20-\mathrm{C} 15-\mathrm{C} 16-\mathrm{C} 17$ & $-4.2(7)$ \\
\hline $\mathrm{C} 4-\mathrm{C} 5-\mathrm{C} 6-\mathrm{C} 7$ & $2.3(8)$ & $\mathrm{C} 15-\mathrm{C} 16-\mathrm{C} 17-\mathrm{C} 18$ & $2.2(8)$ \\
\hline
\end{tabular}




$\begin{array}{llll}\mathrm{C} 5-\mathrm{C} 6-\mathrm{C} 7-\mathrm{C} 8 & 2.4(7) & \mathrm{C} 16-\mathrm{C} 17-\mathrm{C} 18-\mathrm{C} 19 & 1.1(8) \\ \mathrm{C} 6-\mathrm{C} 7-\mathrm{C} 8-\mathrm{C} 9 & -3.5(7) & \mathrm{C} 17-\mathrm{C} 18-\mathrm{C} 19-\mathrm{C} 20 & -2.1(7) \\ \mathrm{C} 6-\mathrm{C} 7-\mathrm{C} 8-\mathrm{P} 1 & -179.3(4) & \mathrm{C} 17-\mathrm{C} 18-\mathrm{C} 19-\mathrm{P} 2 & -173.6(4) \\ \mathrm{C} 11-\mathrm{P} 1-\mathrm{C} 8-\mathrm{C} 7 & -43.4(5) & \mathrm{C} 21-\mathrm{P} 2-\mathrm{C} 19-\mathrm{C} 18 & -39.4(5) \\ \mathrm{C} 10-\mathrm{P} 1-\mathrm{C} 8-\mathrm{C} 7 & 68.3(4) & \mathrm{C} 22-\mathrm{P} 2-\mathrm{C} 19-\mathrm{C} 18 & 73.3(5) \\ \mathrm{P} 1-\mathrm{P} 1-\mathrm{C} 8-\mathrm{C} 7 & -168.5(4) & \mathrm{P} 1-\mathrm{P} 2-\mathrm{C} 19-\mathrm{C} 18 & -167.0(4) \\ \mathrm{C} 11-\mathrm{P} 1-\mathrm{C} 8-\mathrm{C} 9 & 140.6(4) & \mathrm{C} 21-\mathrm{P} 2-\mathrm{C} 19-\mathrm{C} 20 & 148.8(4) \\ \mathrm{C} 10-\mathrm{P} 1-\mathrm{C} 8-\mathrm{C} 9 & -107.7(4) & \mathrm{C} 22-\mathrm{P} 2-\mathrm{C} 19-\mathrm{C} 20 & -98.5(4) \\ \mathrm{P} 1-\mathrm{P} 1-\mathrm{C} 8-\mathrm{C} 9 & 15.5(3) & \mathrm{P} 1-\mathrm{P} 2-\mathrm{C} 19-\mathrm{C} 20 & 21.2(4) \\ \mathrm{C} 1-\mathrm{N} 1-\mathrm{C} 9-\mathrm{C} 8 & 165.5(4) & \mathrm{C} 12-\mathrm{N} 2-\mathrm{C} 20-\mathrm{C} 19 & 166.2(4) \\ \mathrm{P} 1-\mathrm{N} 1-\mathrm{C} 9-\mathrm{C} 8 & -18.9(5) & \mathrm{P} 1-\mathrm{N} 2-\mathrm{C} 20-\mathrm{C} 19 & -19.0(5) \\ \mathrm{C} 1-\mathrm{N} 1-\mathrm{C} 9-\mathrm{C} 4 & -10.1(7) & \mathrm{C} 12-\mathrm{N} 2-\mathrm{C} 20-\mathrm{C} 15 & -9.3(7) \\ \mathrm{Pt} 1-\mathrm{N} 1-\mathrm{C} 9-\mathrm{C} 4 & 165.5(3) & \mathrm{P} 1-\mathrm{N} 2-\mathrm{C} 20-\mathrm{C} 15 & 165.4(3) \\ \mathrm{C} 7-\mathrm{C} 8-\mathrm{C} 9-\mathrm{N} 1 & -175.6(4) & \mathrm{C} 18-\mathrm{C} 19-\mathrm{C} 20-\mathrm{N} 2 & -175.7(4) \\ \mathrm{P} 1-\mathrm{C} 8-\mathrm{C} 9-\mathrm{N} 1 & \mathrm{P} 2-\mathrm{C} 19-\mathrm{C} 20-\mathrm{N} 2 & -3.3(6) \\ \mathrm{C} 7-\mathrm{C} 8-\mathrm{C} 9-\mathrm{C} 4 & -0.1(7) & \mathrm{C} 18-\mathrm{C} 19-\mathrm{C} 20-\mathrm{C} 15 & -0.1(7) \\ \mathrm{P} 1-\mathrm{C} 8-\mathrm{C} 9-\mathrm{C} 4 & 176.2(3) & \mathrm{P} 2-\mathrm{C} 19-\mathrm{C} 20-\mathrm{C} 15 & 172.3(4) \\ \mathrm{C} 3-\mathrm{C} 4-\mathrm{C} 9-\mathrm{N} 1 & 3.1(7) & \mathrm{C} 14-\mathrm{C} 15-\mathrm{C} 20-\mathrm{N} 2 & 2.0(7) \\ \mathrm{C} 5-\mathrm{C} 4-\mathrm{C} 9-\mathrm{N} 1 & -179.9(4) & \mathrm{C} 16-\mathrm{C} 15-\mathrm{C} 20-\mathrm{N} 2 & 178.6(4) \\ \mathrm{C} 3-\mathrm{C} 4-\mathrm{C} 9-\mathrm{C} 8 & -172.4(5) & \mathrm{C} 14-\mathrm{C} 15-\mathrm{C} 20-\mathrm{C} 19 & -173.5(4) \\ \mathrm{C} 5-\mathrm{C} 4-\mathrm{C} 9-\mathrm{C} 8 & 4.6(7) & \mathrm{C} 16-\mathrm{C} 15-\mathrm{C} 20-\mathrm{C} 19 & 3.2(7)\end{array}$

